\title{
Remarques sur la signification des expériences en géodynamique karstique
}

Par Ph. RENAULT

Avec 8 figures dans le texte

\section{RÉSUMÉ}

Distinction entre l'expérience qui «démontre»-ayant valeur d'argumentation - et l'expérience qui «interroge» la nature afin d'isoler un facteur et de préciser la nature de l'action de ce facteur. Concept d'expérience en géologie et en géodynamique. Distinction entre la géodynamique et la physique du globe.

La géodynamique karstique considère l'action de fluides - principalement liquides - sur une roche soluble. Elle se situe à l'intersection des branches géochimie, hydrologie, mécanique des roches de la physique du globe. Dans ses recherches elle utilise les campagnes de mesures dans la nature, éventuellement le montage d'un laboratoire souterrain. Au laboratoire elle dépasse à peine le stade de l'expérience-démonstration. A titre d'exemple sont énumérées une série d'expérience simples, utilisant l'attaque d'une roche soluble par un acide dilué, ceci afin de sérier les problèmes majeurs de la corrosion karstique. Un dernier paragraphe considère les équilibres bicarbonate de $\mathrm{Ca}-\mathrm{CO}_{2}$.

L'expérience fournit le critère empirique à la faveur duquel se constitue la théorie scientifique. Chaque discipline suit un marche méthodologique imposée par l'objet étudié et le critère expérimental sera de nature différente pour chacune d'elles, surtout dans le cas des phénomènes se développant à une échelle sans commune mesure avec les dimensions humaines, dans l'espace (astronomie) ou dans le temps (géologie), et où l'expérimentation «instrumentale» est très limitée dans ses possibilités.

Après un bref rappel des principes de la démarche expérimentale et un historique des expériences tentées par les géodynamiciens (tectonique, géomorphologie, etc...) nous analyserons plusieurs méthodes d'investigations, et en nous appuyant surl'exemple précis de la corrosion karstique, nous verrons la valeur qu'il est possible de leur accorder sur le plan de la connaissance karstologique. 


\section{La notion d'expérience}

Les controverses résultant souvent de la confusion entre plusieurs significations différentes du mème terme, nous débuterons par une définition.

Vouloir rappeler les principes de la méthode expérimentale est ambitieux. Sans évoquer les illustres personnalités qui ont fait connaitre les notions classiques, il faut signaler que, postérieurement, de nombreux ouvrages ont apporté des points de vue originaux, inspirés par les techniques récentes, la cybernétique par exemple (Couffignal).

Nous limitant à un point de vue psychologique élémentaire, nous rappellerons que dans son acceptation empirique, le terme expérience possède deux significations différentes qui, pour se recouvrir partiellement, n'en désignent pas moins deux ordres de réalités qui s'opposent. D'après le classique dictionnaire Larousse, l'expérience a pour but de "démontrer ou de vérifier une chose».

\section{$1^{\circ}$ - L'expérience démonstration:}

Il s'agit de l'opération réalisée par le professeur de chimie provoquant volontairement (ou pas) une explosion, par le camelot cherchant à vendre un produit ou par le prestidigitateur immobilisant un public attentif.

A partir d'un montage de présentation, d'une mise en scène, se crée un «suspense» qui fixe l'attention. Le résultat de l'expérience provoque le «choc» qui engourdit l'esprit par une réaction à la fois de surprise mème lorsque l'on connait d'avance le résultat à obtenir - et de détente, la présentation ayant créé une tension chez le spectateur.

Il convient de ne pas oublier que l'expérience, en tant que démonstration, relève de ce processus inconscient, et que l'engouement $\mathrm{du}$ public pour les choses de science repose sur cet élément spectaculaire des réalisations techniques modernes.

\section{$2^{\circ}$ - L'expérience vérification:}

D'après Lalande, dans le «Vocabulaire technique et critique de la philosophie», «une expérience est le fait de provoquer, en partant de certaines conditions bien déterminées, une observation telle que le résultat de cette observation, qui ne peut être assigné d'avance, soit propre à faire connaitre la nature ou la loi du phénomène étudié». ( $8^{\circ}$ éd., 1960, p. 428). Le souci d'argumentation démonstrative est remplacé ici par une attitude de recherche.

Cette définition classique peut s'exprimer en termes d'action cybernétique (fig. 1) ce qui nous conduit à distinguer les éléments expréimentaux suivants: 


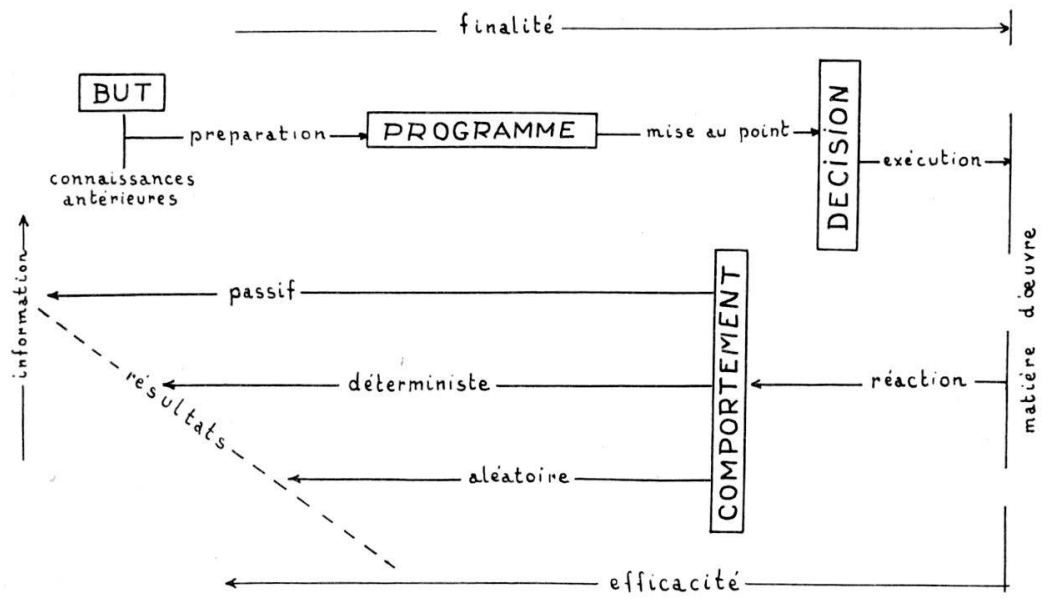

Fig. 1: Schéma de l'acte expérimental. - A partir d'un but (hypothèse) initial, l'élaboration du programme suivi d'une mise au point pratique permet d'agir sur un équilibre naturel (matière d'œuvre). Les réactions correspondent à un comportement passif (conforme au programme), détermiste (non conforme au programme, mais avec des variations explicables par les lois connues), aléatoire (indépendante du programme). Les résultats, incorporés au corps de doctrine conçernant le domaine d'action envisagé, seront utilisés pour fixer de nouveaux buts de recherches.

- le but exprimé dans l'hypothèse,

- le programme,

- l'exécution conduisant à une observation,

- l'efficacité de cette observation qui, traduite en termes d'information, permet d'élaborer un modèle de structure analogique: la théorie.

a - But: L'expérience vérifie une hypothèse. Elle ne peut apporter de faits bruts. Au stade de la «Science qui se fait», le chercheur, dans une certaine perspective, examine plusieurs phénomènes constatés. Pour les "comprendre», il est amené à créer une interprétation «à priori», par assemblage de concepts articulés suivant une démarche logique. Cet assemblage est modelé par le langage servant de support à l'activité intellectuelle et cette construction verbale «à priori» doit être confrontée avec le réel.

Tous éléments bien précisés par Lalande; l'hypothèse est une "proposition reçue, sans égard à la question de savoir si elle est vraie ou fausse», mais elle n'est hypothèse que parce qu'elle est «destinée à être ultérieurement vérifiée». Hors du domaine de la vérification scientifique, l'hypothèse devient un outil de travail philosophique, 
simplement une conjecture. Cette nécessité de vérification définit la méthode expérimentale.

En d'autres termes l'hypothèse concrétise un doute, obligeant l'expérimentateur à suspendre son jugement jusqu'après vérification.

b - Ce qui conduit à formuler un programme d'expérience, autrement dit à préciser par quel biais les problèmes (le doute) impliqués par l'hypothèse pourront être résolus. Sur le plan réalisation cette préparation est déjà importante: «il peut suffire de poser le programme d'une expérience... sans qu'il soit nécessaire de la réaliser pour en tirer ... déjà ... certaines conclusions $\left.»^{1}\right)$. Ce point de vue, négligé dans les manuels, s'avère d'autant plus important que l'ampleur des moyens de recherches modernes augmente, qu'il s'agisse d'expériences coûteuse (physique atomique, réalisations de travaux publics) ou du recours à des machines analogiques du type ordinateur.

La réalisation du programme exige la mise en œuvre de moyens variés, perfectibles en cours de réalisation. Il est nécessaire de rappeler que l'un des caractères essentiels de l'expérience est sa reproductibilité. Dans certains cas les résultats n'ont été obtenus qu'une fois, l'opérateur ignorant les éléments qui furent déterminants dans sa réussite.

c-L'expérience a pour but de provoquer une observation. La question des rapports entre observation et expérience est un exposé classique de la littérature philosophique; certains ont voulu opposer ces deux termes, l'observation se limitant à constater, alors que, dans l'expérience, le fait est provoqué. La réalité est plus subtile: «'observation scientifique est toujours la réponse à une question précise (Bergson).»

La nature de cette réponse et la façon de poser la question varient considérablement suivant les sciences. Parfois, l'expérience se limite à un contrôle par observation directe. La découverte de Neptune par le Verrier, en astronomie, constitue une démarche de ce type. De façon analogue la géologie parait se limiter à un simple relevé d'échantillons au hasard des affleurements. En réalité le géologue suit une marche expérimentale en posant une hypothèse de structure et la vérifiant par examen d'affleurements en un certains nombres de points.

Dans d'autres cas, en physique par exemple, l'observation sera possible grâce à un montage artificiel. Il serait alors possible de parler d'expérimentation instrumentale.

d - Les réponses obtenues, c'est à dire les réactions de la matière d'œuvre (fig. 1) à la sollicitation provoquée possèdent une signification en fonction du but fixé par l'hypothèse.

\footnotetext{
1) Goguel, J., 1948, p. 500.
} 
Les résultats pourront être de nature différente: passifs, c'est à dire conformes aux résultats prévus par le programme, déterministes, c'est-à-dire non identiques aux résultats présumés, les variations étant explicables en tenant compte de lois connues, qu'il faudra faire intervenir dans l'élaboration d'un nouveau programme, aléatoires ou indépendants du programme.

Les premières hypothèses formulées ont un caractère "stratégique» c'est-à-dire conçernent l'ensemble du problème étudié. Les résultats "déterministes" soulèvent des problèmes secondaires conduisant à la formulation d'hypothèses tactiques dont la vérification est nécessaire à la solution du problème global initial.

L'ensemble des résultats, recoupés et contrôlés, interprétés suivant la nature du résultat se transforme en information, c'est-à-dire que ces résultats ont une valeur de connaissance relativement au milieu testé. Ces informations seront classées à l'intérieur d'une théorie, avec généralisation s'appuyant sur une référence aux connaissances antérieures. Elle représente un «état actuel» de notre connaissance du domaine étudie.

\section{Evolution de l'idée d'expérience en géodynamique}

$$
1^{\circ} \text { - Géologie, géodynamique et physique du globe }
$$

La géologie au s.s., est une science historique au s.l. qui a pour but de retracer les phases successives de l'évolution de l'écorce terrestre et de les dater. Elle se fonde sur les principes de superposition et de l'uniformitarisme. Nous renvoyons aux traités classiques.

La géodynamique étudie les mécanismes de cette évolution. Elle utilise les résultats de sciences analysant le moment actuel de cette histoire : pédologie, océanographie, etc.... et des sciences qui fondent les précédentes: biologie des sols, mécanique des sols, etc.... Mais lorsque l'on envisage l'ensemble de l'évolution géologique il est nécessaire d'imaginer des phénomènes complètements différents de ceux qui relèvent de l'observation à l'échelle humaine. Leur dimension est cosmique, et il est impossible d'avoir une vue d'ensemble de leur disposition dans l'espace - celui-ci étant partiellement inaccessible et dans le temps. Ces mécanismes ne peuvent être reconstitués que par des opérations mentales s'appuyant sur une mosaïque d'observations partielles et qui aboutissent cependant à des enchaînements cinématiques ${ }^{2}$ ) - traduisant une conception géologique - se transformant en explication dynamique - mais requerrant alors de nombreuses hypo-

2) Voir par ex. Auboin - 1961. 
thèses qui, lorsque celles ci sont invérifiables, laissent la question pendante et menacée par le dogmatisme.

Du point de vue expérimental, nous distinguerons :

a - la géologie s.s. comme nous l'avons signalé plus haut, procède d'une démarche qui obéit aux principes de la méthode expérimentale, mais son caractère historique (Conte) la met en dehors de l'expérimentation instrumentale.

b - la physique du globe appliquant les résultats de la mécanique des fluides, de la rhéologie, etc... à l'écorce terrestre actuelle. La notion de temps impliquée dans ses travaux est une durée, le paramètre $\mathrm{t}$ des physiciens, et les modifications observées correspondent aux variations d'un équilibre en fonction de $t$,

c-la géodynamique qui reconstitue les mécanismes d'évolution anciens en les étudiant à des échelles variées, mais le plus souvent d'ordre géologique, c'est-à-dire très différentes de l'échelle humaine. Elle envisage, non plus des durées, mais des successions de phénomènes, définis en fonction d'une chronologie relative. L'aspect le plus important de ce «temps historique» est que chaque événement émerge d'un état antérieur comportant nécessairement un certain nombre de caractères qui orientent le mouvement général dans un sens et non pas dans un autre.

La géodynamique présente donc plusieurs éléments d'incertitude. Tout d'abord il existe inévitablement des lacunes dans les reconstitutions. De ce fait la géodynamique risque de mettre sur le même plan divers effets se classant à des niveaux différents de l'échelle chronologique ou causale, ce qui crée, de façon plus ou moins temporaire, des oppositions irréductibles entre le fait géodynamique et la physique du globe.

Ces incertitudes rendent l'explication incertaine. «Expliquer c'est présenter l'ordre dans le présent comme une conséquence de l'interaction dans le passé» (Reichenbach, cit. Costa p. 42). Il existe une opposition foncière entre la causalité temporelle des physiciens aboutissant à une prédiction, et la reconstitution des causes effectuée à partir d'une analyse des effets. "Il ne faut pas oublier lorsque nos ensembles sont choisis pour représenter les probabilités des événements dans le monde réel, que, si les probabilités des événements subséquents peuvent être souvent déduits de celles des événements antérieurs, il est bien rare que les probabilités des événements antérieurs puissent être déduites de celles des événements subséquents, car il est rare qu'on ait le droit d'exclure la considération de la probabilité antécédente des événements antérieurs" (Gibbs, cit. Costa). 


\section{$2^{\circ}$ - Esquisse historique}

Géologie, physique du globe et géodynamique se sont développées parallèlement. Un bref historique permettra de comprendre cette évolution.

"Il y a près de 200 ans M. de Buffon dans ses forges de Montbard fit chauffer au rouge une série de boulets de tailles échelonnées depuis $1 / 2$ pouce jusqu'à 6 pouces, puis il nota le temps au bout duquel chacun d'eux était suffisamment refroidi pour qu'on puisse le tenir dans la main sans se brûler. Extrapolant la loi linéaire qu'il avait ainsi mise en évidence jusqu'aux dimensions de la terre, il en déduisit pour l'âge de celle-ci après diverses corrections, le chiffre de 63000 ans. L'important, dans cette expérience, était le principe qu'elle posait, que l'histoire de la terre et son origine relevait de la physique» (Goguel, 1961).

En 1788 James Hall réalise les premières expériences tectoniques en comprimant latéralement un empilement de drap, puis un empilement de lits d'argile. "Il est à peine besoin de souligner l'importance d'une telle expérience à l'époque où elle fut réalisée. En montrant la possibilité de rapporter les plis à des compressions latérales, elle a ouvert à la tectonique une voie demeurée féconde»33).

$\mathrm{Au}$ cours du $\mathrm{XIX}^{\circ}$ siècle les tentatives expérimentales se poursuivent. De la Noë et Margerie analysent les lois de l'érosion fluviale en faisant circuler un petit écoulement sur un empilement de couches de dureté inégales $\left.{ }^{4}\right)$. D'Estrem effectue quelques expériences montrant que la chaleur peut fragmenter une roche et donner naissance à une brèche. Richard note la formation de pisolithes dans une machine à vapeur. Tassy étudie l'encroûtement calcaire d'une canalisation. Etc....

C'est avec Daubrée que s'affirme une «géologie expérimentale». En 1859 il prouve que la chaleur seule ne suffit pas à expliquer ] métamorphisme et que l'eau doit être considérée comme l'agent essentiel de ce phénomène. En tordant une plaque de verre il provoque l'apparition de fissures, qu'il assimile aux diaclases. Il étudie artificiellement la schistosité et les déformations concomitantes des fossiles, le striage des roches et le façonnement des galets, le résultat de l'échauffement des argiles, le rôle morphologique ou chimique des gaz souterrains à haute pression et haute température (en faisant éclater

$\left.{ }^{3}\right)$ Pour un historique de la tectonique expérimentale nous renvoyons à Goguel J. - 1948 p. 498-516.

4) Se référer à la bibliographie en annexe à ce texte. 
des cartouches de dynamite). Nous avons compté 117 références de publications consacrées par lui à ces expériences, tous ces travaux étant rassemblés dans les deux gros volumes parus en 1879 sous le titre d'«Etudes synthétiques de géologie expérimentale».

Cette voie sera poursuivie par Stanislas Neunier qui, dans les laboratoires du Museum d'Histoire Naturelle de Paris, à la fin du $\mathrm{XIX}^{\circ}$ siècle réalise in vitro les phénomènes les plus variés: cristallisations intra sédimentaires, galets striés, cheminées de fées, dendrites de manganèses et surtout cavernes articielles en dirigeant un jet d'eau acidulé sur une dalle calcaire.

A la même époque citons encore Fayol rendu célèbres par les expériences destinées à étayer sa théorie allochtone de la genèse des bassins houillers. Plus récemment il faut mentionner le volcanisme expérimental de Belot (fig. 2), les expériences sur marmites de géant de Alexander, etc....

Mais, depuis le début du siècle, ces tentatives se raréfient. Dans le cadre des sciences de la terre, le point de vue expérimental s'est transformé. En effet la connaissance rationnelle suit une démarche immuable. Elle évolue de l'expérience sensible vers l'abstraction, et tout progrès se ramène à la prise en considération de concepts antérieurement sous jacents aux données de l'expérience commune. «Les lois générales de la physique ont presque toujours été pressenties, démontrées et étudiées à propos de questions particulières à la physique du globe avant qu'il puisse en être donné une expression générale; Pascal a établi la notion de pression d'un gaz en même temps qu'il étudiait la pression atmosphérique...» (Goguel, 1959).

La géomorphologie et la géodynamique, par un certain nombre de tentatives expérimentales, maintenant anciennes, ont conduit au développement de branches complexes de la physique moderne, qui le plus souvent se décomposent en plusieurs disciplines parallèles, les unes éminemment abstraites et recherchant des résultats de valeur hautement générales, les autres, plus techniques, étudiant les applications de ces résultats généraux à la complexité des cas rencontrés dans la nature sauvage (hydraulique appliquée à l'océanographie par exemple) ou bien modifiée par les grands travaux (d'aménagements hydrauliques par exemple).

Sans avoir la prétention d'être complet mentionnons par exemple la rhéologie. Cette science, d'introduction récente, étudie la viscosié, la plasticité, l'élasticité, ou, de façon plus générale, $l^{`}$ écoulement de la matière. Hautement théorique et de forme très mathématique, elle se prolonge dans un certain nombre de branches appliquées, tel que la mécanique des sols. 
Il y a deux cents ans celle ci se résumait en quelques préceptes empiriques transmis par des générations de maçons. Plus tard, dans le cadre fourni par les travaux mathématiques de Coulomb (1773), Boussinesq, etc... quelques notes (Collin, 1846) préludent aux importants développements couronnés par l'œuvre de A. Caquot en France et de K.Terzaghi aux U.S.A. Plus récemment se développe une «mécanique des roches» portant sur un objet légèrement différent (Talobre).

Ces mécaniques spéciales retrouvent la géomorphologie avec les récents travaux d'Avenard, sur l'équilibre des versants de vallée, ou l'œuvre magistrale de J. Goguel conçernant la mécanique des déformations de l'écoree terrestre (1948).

Toujours dans le domaine rhéologique, mentionnons les expériences de déformation de roches ou de cristaux soumis à des pressions de plusieurs tonnes $/ \mathrm{cm}^{2}$. Là, en début de siècle, Bridgman fut un initiateur. Plus récents citons les travaux de Griggs. Ceux- ci introduisent aux expériences de synthèse minéralogique à haute pression et haute température, par exemple, les expériences de fonte anatectique de Winkler aboutissant à reproduire les mécanismes du métamorphisme.

Ce qui nous conduit à la géochimie, science subdivisée en plusieurs branches qui prolongent les grands chapitres de la pétrographie. Il existe ainsi une géochimie des silicates et des minerais, conçernant directement la géodynamique interne. De la même façon, en rapport avec la pétrographie sédimentaire, il existe une géochimie de la partie externe de l'écorce terrestre. L'une de ses sources peut être cherchée dans la chimie agricole, illustrée par Chloesing, Muntz, etc.... vers la fin du XIXe, celle-ci s'épanouissant dans la pédologie qui a pris naissance en Russie au $\mathrm{XX}^{\circ}$ siècle. Parallèlement se développe la géochimie des carbonates, des eaux souterraines, etc.... présentant un caractère original: dans ce domaine les données biologiques se superposent à la physico-chimie. La cristallogenèse est prise en considération par toute une école et il faut souligner l'importance des néoformations argileuses (Godard, 1963) dans le problème complexe de la décomposition des silicates.

De la même façon la pédologie considère attentivement certaines questions propres à la mécanique des sols; citons les processus thermoclastiques, cryoclastiques, les éclatements par cristallogenèse.

Pour rester dans le domaine de la mécanique, mentionnons les implications géophysiques de l'hydrodynamique.

Les premières observations sur les «Fontaines» faites par Mariotte (1700), Darcy (1856) ont précédé le développement de l'hydraulique souterraine guidant les mesures en hydrogéologie. 
Les expériences de Du Buhat (1786), Dupuit, etc.... annoncent les travaux de Gilbert (1914) et de l'actuelle hydraulique fluviale (Leliavsky) étudiant le transport des alluvions et le façonnement des lits de rivière. Ce qui introduit aux expériences de sédimentation de Berthois, Kuenen, etc.... qui reprennent les travaux anciens de Daubré, Fayol, Meunier (exposés dans Cayeux, 1929) sur le façonnement des galets et des grains de sable, mais en étudiant la marche du phénomène à l'aide d'indices numériques permettant une définition quantitative du processus.

Nous arrêterons là cette énumération limitée à quelques branches intéressant directement la karstologie.

$$
\begin{gathered}
3^{\circ} \text { - Rapports de la physique du globe } \\
\text { et de la géodynamique }
\end{gathered}
$$

Dans quelle mesure la physique du globe contribue-t-elle au progrès de la géodynamique? En d'autres termes il faut préciser les limites d'application de la physique du globe.

La géologie, c'est-à-dire les enchainements historiques reconstitués à partir des observations de terrain, suppose un certain nombre de mécanismes géodynamiques qui doivent ètre envisagés avec les réserves esquissèes plus haut (incertitude de la reconstitution des causes). La reconstitution géodynamique présente un caractère essentiel: elle repose toujours sur l'examen d'une structure ou d'une disposition particulière et toute généralisation ou formulation de loi résulte simplement de la comparaison d'un nombre plus ou moins grand de cas particuliers. L'induction géodynamique se limite à une dialectique des éléments communs aux structures comparées.

A l'opposé, la rationnalité de la physique du globe est de type mathématique. La compréhension des phénomènes analysés impose le recours aux données chiffrées: considérant un phénomène naturel dans sa complexité - le débit d'une rivière ou le système de contraintes régnant sous un plateau - elle tente de comparer des mesures à un modèle, non plus empirique mais axiomatique, présenté sous une forme mathématique, et faisant appel aux dernières ressources de cette technique.

Mais l'écorce terrestre est un domaine complexe, et son analyse impose le recours a un certain nombre d'hypothèses simplificatrices. Cette nécessité conduit à une double démarche, analytique et empirique.

La démarche analytique prolonge la rationnalité ci-dessus définie. Par exemple l'hydraulicien s'attachera à déterminer les forces et les 
divers paramètres qui maintiennent une particule en suspension dans un écoulement. Ces considérations conduisent à un enchainement totalement logique. Citons les études portant sur le transport des sédiments, l'hydraulique souterraine ou bien l'ouvrage récent de Scheidegger.

Il s'agit de résultats brillants mais qui se situent à un degré encore élevé de généralités. L'application à un problème complexe est très vite arrêtée. Dans le cas de la mécanique de l'écorce terrestre: «En cherchant les lois qui régissent la répartition des contraintes dans un ensemble de roches variées, et la déformation ultérieure de celles-ci, nous avons constaté que les équations aux dérivées partielles qui représenteraient ces phénomènes sont tellement compliquées qu'il n'y a aucun espoir de pouvoir les intégrer avec les conditions aux limites qui correspondent à un problème réel (Goguel, 1948, p. 498).»

La démarche analytique est donc limitée par les incertitudes nées de la complexité du domaine envisagé. Il est alors possible de faire appel au traitement analogique. Les lois de l'hydraulique ne permettent pas de prévoir la géométrie d'un lit fluvial, mais à partir d'un traitement théorique reposant sur la notion d'entropie exprimée en termes de probabilité d'états variés, Leopold et Langbein, dans un travail très remarqué, sont arrivé à déduire le profil d'équilibre théorique d'un cours d'eau. Il est possible ainsi de définir les limites d'un éventail de possibilités.

En se rapprochant d'une analyse encore plus poussée des phénomènes actuels l'emploi d'une méthode empirique s'impose alors: observation continue au cours de campagnes d'observations systématiques ou de réalisations artificielles, ou bien recours aux modèles réduits.

Pour conclure nous renverrons d'abord au tableau ci joint groupant les caractères comparés de la géodynamique et de la physique du globe. Pour schématiser, nous dirons simplement que le géodynamicien se trouve dans la situation du garagiste cherchant à dépanner un moteur dont il ignore à peu près tout; tandis que le physicien du globe ressemble à l'ingénieur construisant un moteur qui doit satisfaire à certaines conditions. Le premier fait un large appel à l'intuition et aux comparaisons. Il procède qualitativement. Le second prévois et calcule, ce qui ne l'empêche pas d'effectuer de nombreux essais.

Il est dans l'ordre des choses que la géodynamique se rapproche de la physique du globe: par exemple mentionnons la mécanique de l'écorce terrestre qui fait le lien entre la tectonique et les sciences qui étudient l'intérieur de notre globe (séismologie, gravimétrie, etc....). Cet idéal se réalise de deux façons. La physique du globe a la possibilité 
de préciser l'âge des successions par la mise au point de «chronomètres» fournissant des datations en valeur absolue (rapports isotopiques du plomb, de l'ionium, du carbone, etc....). Elle apporte en outre des renseignements quantitatifs sur les mécanismes naturels impliqués par les reconstitutions géodynamiques. Le dialogue est maintenant établi entre la géodynamique qui pose des problèmes et la physique du globe qui discutte et valide des hypothèses.

\begin{tabular}{lll}
\hline & \multicolumn{1}{c}{ Géodynamique } & \multicolumn{1}{c}{ Physique du globe } \\
\hline But & Phénomènes géologiques & Phénomènes actuels \\
Démarche & $\begin{array}{l}\text { Reconstitution des causes } \\
\text { à partir d'effets constatés } \\
\text { non reproductibles }\end{array}$ & $\begin{array}{l}\text { Prévision d'effets à partir } \\
\text { des variations paramétri- } \\
\text { quesd'unesituation initiale }\end{array}$ \\
Méthode & $\begin{array}{l}\text { Comparaison de cas parti- } \\
\text { culiers; recherche des élé- } \\
\text { ments communs }\end{array}$ & Analyse axiomatique \\
Temps & $\begin{array}{l}\text { Reconstitution } \\
\text { d'enchainements }\end{array}$ & Durée constatée \\
Progression & Du complexe au simple & Du simple au complexe \\
Données & Qualitatives (possibilités) & Quantitatives (précision)
\end{tabular}

\section{Opérations expérimentales en géodynamique}

Pour préciser les caractères de l'expérimentation instrumentale en géodynamique karstique, il est d'abord nécessaire, à la lumière des notions précédemment exposées, de définir les opérations réalisables.

$$
1^{\circ}-\mathrm{Au} \mathrm{laboratoire}
$$

Nous distinguerons 3 opérations principales, différentes dans leur objet et leur destination:

A - La mesure, ou observation quantitative, est indispensable dans l'étude d'un milieu continu où les particularités ne peuvent être définies qualitativement, ou bien ne sont pas identifiables par la simple observation macroscopique et nécessitent un appareillage spécial. Le domaine en est varié, allant des expériences de Griggs (mesure des déformations sous haute pression) aux analyses sédimentologiques. 
La réalisation des mesures pose un certain nombre de problèmes, d'ordre méthodologique, métrologique (précision), statistique (signification des mesures, contrôle, exploitation).

B - La démonstration, est une forme tout à fait banale de l'expérimentation, qu'elle soit originale (fig. 2) ou réalisée spontanément dans un montage industriel (vagues d'érosion observées sur un rotor de pompe par exemple). Ces expériences ont joué un rôle important à certaines époques. Non pas qu'elles prouvent quelque chose: une couche d'argile comprimée (fig. 2) «ne ressemble pas plus à une montagne qu'une tôle ondulée à la chaîne du Jura" (Roubault), mais elles attirent l'attention sur la possibilité d'un mécanisme géodynamique.

Souvent naïves, elles «servent surtout à concrétiser l'hypothèse qu'elles sont destinées à illustrer... Elles n'apportent rien qui ne soit implicitement supposé par le mécanisme mis en œuvre... On peut presque dire qu'il est possible de réaliser une expérience venant à l'appui de n'importe quelle théorie géologique, même complètement inexacte» (Goguel, 1952, p. 199).

C - L'observation sur modèle-réduit est une combinaison des deux cas précédents: il s'agit d'une expérience s.s. où l'on cherche à reconstituer un phénomène naturel, le déroulement de l'expérience étant accompagnée de mesures donnant une signification quantitative aux résultats obtenus.

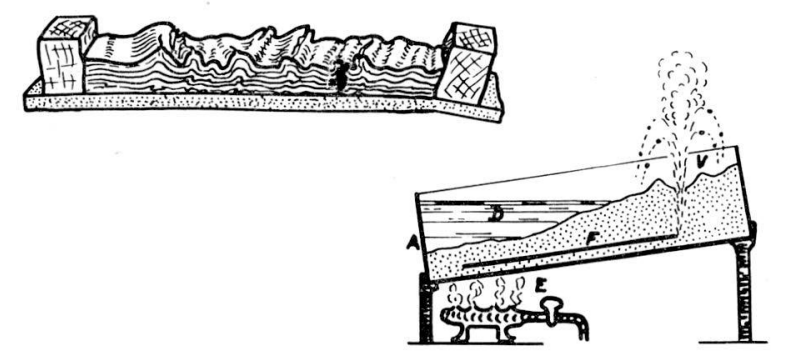

Fig. 2. Expériences «démonstrations» de géologie expérimentale. - En haut: expérience de plissement tectonique sur modèle: argile disposée entre deux butées sur une bande de caoutchouc tendue. Lorsque le caoutchouc se relache l'argile se plisse (Goguel, 1952).- En bas : volcanisme expérimental de Belot. - Dans un bac de tôle A une certaine quantitée de sable (en pointillé) imbibé d'eau (D) ennoie une ardoise (F) parallèle au fond. La chaleur du réchaud (E) porte à ébullition l'eau contenue dans le sable et la vapeur s'échappe vers le "continent» sous la plaque F en formant un cratère (V) tandis que l'eau du bassin reste froide (d'après Science et Vie, $\mathrm{n}^{\circ} 40$, janvier 1920). 
La réalisation s'effectue en respectant certaines règles dites de similitudes. En effet les phénomènes étudiés se développent dans la nature à une échelle très différente de celle que nous pouvons réaliser in-vitro. Il sera donc nécessaire de réduire les dimensions. Cette réduction ne peut s'effectuer au hasard: il faudra réduire longueurs, masses spécifiques, vitesses, temps, etc... en respectant diverses conditions, relations existant entre les forces mises en présence et dont le rapport ne doit pas varier si l'on désire que le modèle soit «homologue» du phénomène qu'il est sensé représenter.

En hydraulique, il existe 4 conditions (de Mach, Reech et Froude, Reynolds, etc...) reliant dimensions, vitesse, viscosité, etc... Deux de ces échelles étant choisies librement, les 4 autres s'en déduisent à partir des conditions de similitudes.

Il est évident que subsiste un effet d'échelle, la similitude ne pouvant être respectée jusque dans les détails. L'interprétation des mesures réalisées est done limitée par les risques d'erreurs suivants:

a - une correction, insignifiante au laboratoire, devient déterminante à une échelle supérieure (Carey);

$\mathrm{b}$ - des paramètres interdépendants, varient indépendamment avec l'accroissement des longueurs ou du temps (Carey);

c-l'existence de seuils physiques limite les possibilités d'extrapolation (Carey);

d-l'évolution naturelle comprend, entre les phases de transformation, de nombreux épisodes de stabilité, susceptibles de jouer un rôle dans le développement du phénomène, et qui se trouvent éliminés dans une reconstitution expérimentale (Birot, 1962);

e - les équilibres naturels sont réglés par des effets réciproques (feed-back) agissant de deux façons: 1. en stabilisant: un facteur tendant à détruire un équilibre est compensé par d'autres phénomènes rétablissant cet équilibre ou détruisant l'élément perturbateur, ou 2. en catalysant: l'action du facteur augmente l'activité de l'effet (ex.: creusement d'une doline). Un montage expérimental néglige ou limite considérablement ces rétroactions.

Malgré ces quelques réserves la technique des modèles réduits est très utilisée, surtout en hydraulique. Citons en France, le Laboratoire d'Hydraulique de l'Institut de Mécanique des Fluides de Toulouse, dont la réputation est internationale.

D - La méthode des analogies ou du calcul expérimental analogique est connue depuis que, en 1931, Perès et Malavard ont observé que des phénomènes physiques de nature très différente sont régis par les mêmes équations. Toutes les fois qu'il sera possible de réaliser une 
image électrique de l'équation étudiée, le calcul pourra être remplacé par une mesure. Les problèmes d'élasticité, de mécanique des fluides, etc... pourront se résoudre ainsi. L'Institut Blaise Pascal du C.N.R.S. est spécialisé dans les applications de cette méthode.

Nous signalons cette méthode en raison des possibilités qu'elle offre pour résoudre certains problèmes ayant atteint un degré élevé de formulation théorique. Le travail récent de Léopold et Langbein a valeur d'exemple en ce domaine.

En conclusion il faut distinguer:

$1^{\circ}$ - la mesure qui définit quantitativement un caractère,

$2^{\circ}$ - les opérations de caractère analogique, c'est-à-dire reposant sur une identité de rapports représentant des fonctions communes. L'analogie ne doit pas être confondue avec les identités de formes, ou ressemblances, soumises aux aléas des phénomènes de convergence.

Ce concept d'analogie introduit à la notion de modèle. Quels rapports peut on envisager entre réalité, modèle et théorie? Ce problème est assez important pour qu'un Symposium lui ait été consacré en 1961 (Destouches).

\section{$2^{\circ}-$ Sur le terrain}

Dans un certain nombre de cas il sera possible d'expérimenter directement dans la nature sans avoir recours aux méthodes analogiques. Mentionnons:

A - La campagne de mesures portant sur un ensemble de caractères dont il est possible de suivre les variations dans le temps et dans l'espace. Les possibilités sont nombreuses: campagnes hydrologiques, météorologiques, géochimiques, géophysiques... allant de la mesure effectuée sur place jusqu'au prélévement d'échantillon, en sédimentologie par exemple, pour analyse détaillée au laboratoire.

B-La modification artificielle, une infinie variété pouvant ètre proposée à l'imagination du lecteur. Citons deux cas très différents:

$1^{\circ}$ - Les grands travaux aboutissent à des modifications très poussées du paysage: ainsi la création d'un lac artificiel lors de la construction d'un barrage hydroélectrique. Les conséquences hydrologiques d'une telle réalisation peuvent être instructive. Précisons d'ailleurs, la réalité étant indépendante de nos divisions, que bien avant la mise en eau, les études préliminaires réalisées avec des moyens à l'échelle de l'ouvrage projeté, apportent des renseignements qui, autrement, demeureraient inaccessibles. 
$2^{\circ}$ - Les cavités artificielles (mines, carrières, tunnels, puits, etc...) permettent de contempler certains phénomènes «à l'état pur» avec, en outre, possibilité d'en évaluer la vitesse. Il est possible d'observer ainsi des concrétions de formation accélérée (Meunier, 1902, Perna, 1961, etc...) des vermiculations argileuses (Renault, 1963), des phénomènes d'effondrement, hydrologiques (problèmes d'exhaure), climatiques, etc... Il sera même possible d'étudier une faune cavernicole (Balazuc) caractéristique.

D'autres exemples de réalisation artificielles pourraient être citées. L'aménagement d'une grotte modifie, de façon parfois catastrophique, les conditions naturelles. Les constructions urbaines, en révélant l'existence d'une «maladie des pierres» ont permis l'étude d'un certain type d'altération atmosphérique. Le rôle de la porosité dans la cryoclastie a été miş en évidence par l'étude de la résistance des revètements routiers à l'action du gel.

Un jour prochain les expériences de pluie artificielle permettront peut être d'organiser l'étude hydrogéologique d'une région donnée.

C - L'emploi des témoins et traçeurs est bien connu des spéléologues ayant réalisé des expériences de coloration à la fluorescéine. Les possibilités de la méthode sont très étendues. Mentionnons l'utilisation des traçeurs gazeux (odorant ou colorés) pour suivre le trajet d'une circulation d'air, la technique des marques pour suivre le développement de l'érosion (Rougerie) ou le mouvement d'un glacier, l'étude du transport des sédiments par témoins radioactifs ou fluorescents (Grandclément, Ajbulatov, Ramette). Combinés avec les campagnes de mesures et d'échantillonnage systématique, l'emploi des traçeurs permet d'appuyer une étude cinématique cohérente.

D - Pour conclure nous dirons simplement que cette «expérimentation de terrain" complète le laboratoire sans le remplaçer.

En effet, l'existence d'un phénomène étant établie, la raison d'être du laboratoire est de pouvoir faire varier systématiquement un certain nombre de facteurs, afin d'établir leur modalité d'action respectives.

Dans la nature les conditions sont immuables, ou faiblement modifiées dans le cas d'une réalisation artificielle, et seuls certains paramètres varieront (pluviométrie par exemple), ceci dans des limites relativement étroites.

L'expérimentation de terrain permet de résoudre des cas particuliers. Lorsque le nombre de cas devient assez important, la comparaison statistique (Cazalis) permet de généraliser dans la mesure où 1 , le nombre d'exemples naturels est suffisant, 2 , la dispersion des 
points significative. Mais dans la plupart des cas le nombre des facteurs intervenant oblige à combiner laboratoire et mesures de terrains (par exemple voir Einstein et Krone).

D'une façon générale les études de terrain sont une introduction obligée à l'expérimentation au laboratoire.

\section{Caractères de l'expérimentation en géodynamique karstique}

Dans le cas plus particulier de la dynamique karstique, le développement du phénomène est commandé par l'intervention de fluides (gazeux et surtout liquides) s'exerçant sur une roche soluble (fig. 3). L'analyse des mécanismes de creusement ou d'évolution relève ainsi de la géochimie, mais pas seulement de cette dernière, et il faut tenir compte des éléments définis par la physique du globe (hydrologie et climatologie, mécanique des roches, etc...) et qui conditionnent le fonctionnement de ces processus géochimiques.

Ces données physiques et chimiques étant connues, leur application doit être envisagée dans le cadre d'un massif dont les dimensions s'évaluent en $\mathrm{km}$. A ce stade plus précisément géodynamique et géologique intervient la notion d'échelle du phénomène.

D'autres processus doivent être pris en considération. Ils sont plus accessoires, mais jouent un rôle dans l'évolution d'un karst. Citons par

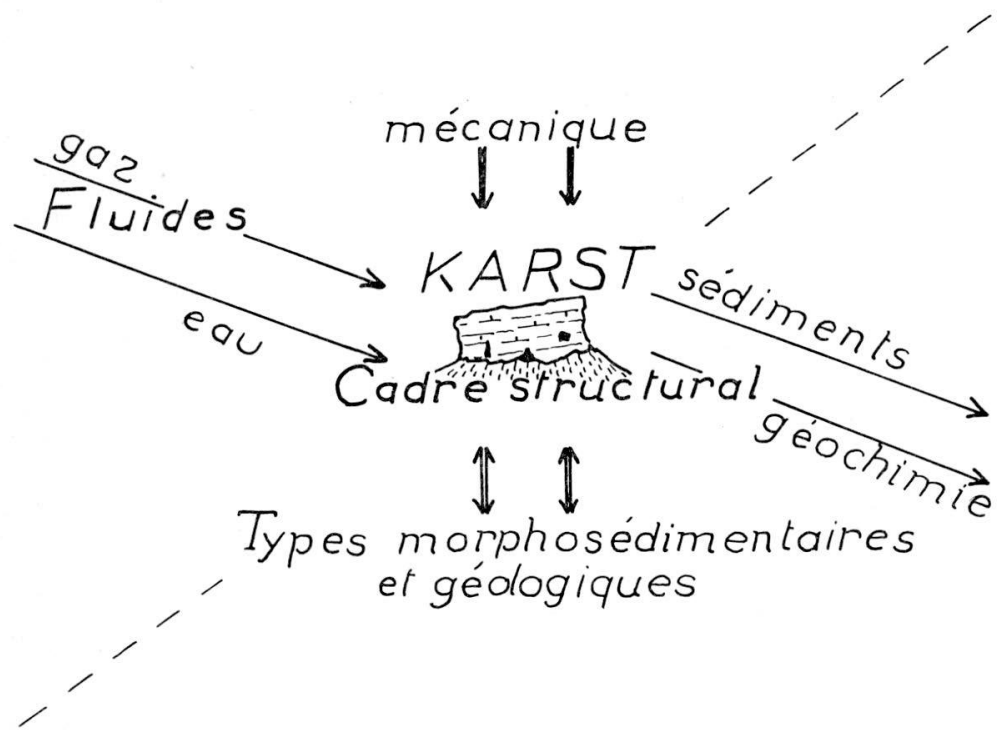

Fig. 3. Schéma du processus karstique. 
exemple les phénomènes de sédimentation argileuse sur paroi (Renault, 1963).

Les grandes lignes de notre sujet étant ainsi traçées, précisons les remarques du chapitre précédent dans le cas particulier de la karstologie et de la spéléologie.

$$
1^{\circ} \text { - Expérimentation sur le terrain }
$$

Les campagnes de mesures (hydrologiques, géochimiques, etc...) constituent la base de l'étude d'un karst donné. En se réfèrant aux publication de J. Corbel et H. Roques, il est facile de voir que, dans le cadre de la géochimie, celles ci conduisent rapidement à des conclusions d'ordre très général: Corbel est ainsi arrivé à un classement climatique des karsts, reposant sur des critères géochimiques.

Il faut dire un mot des laboratoires souterrains et de leurs possibilités expérimentales. Le laboratoire souterrain est constitué par une grotte naturelle ou une cavité artificielle aménagée, pour que l'accès en soit facile, cloturée, pour que l'intrusion de visiteurs intempestifs ne risque pas de troubler les expériences en cours. Les installations sont conçues de façon à perturber au minimum les conditions naturelles (température, humidité, etc....) de la cavité ce qui permet d'entreprendre des observations systématiques, les aménagements permettant de fréquentes visites, et des séries de mesures précises, grâce à l'emploi d'appareils fragiles et encombrant (Renault, 1961).

Les laboratoires souterrains étaient initialement conçus pour effectuer un travail biologique: élevage de cavernicoles, étude de la reproduction et des larves, écologie. Dans le cas du laboratoire souterrain de Moulis (Ariège, France) les buts se sont considérablement élargis, car les problèmes posés par le monde souterrain se tiennent, et il est devenu indispensable de mettre sur pied une «histoire naturelle» des cavernes.

Dans le domaine de la spéléologie physique, les réalisations actuelles portent sur la météorologie (Polli, 1958; Renault, 1961); la croissance des stalactites (Polli, Dor et Hotterbeex) et la géochimie des eaux d'infiltration (Roques). En aménageant des cavités présentant un milieu adéquat, d'autres recherches pourraient ètre envisagées: mesures hygrométriques et évaluations du taux de condensation, mouvements de sédiment dans une rivière souterraine, et, dans une cavité active, variations des pressions de terrains en fonction des saisons et des crues.

Ce genre d'investigation diffère de l'expérimentation biologique sur deux points: 
a - Mobilité - En biologie, pourvue que les conditions climatiques se trouvent respectées, il sera toujours possible de conserver des cavernicoles dans l'obscurité, qu'il s'agisse d'une grotte ou d'une chambre climatisée. Par contre la recherche du lieu propre à certaines observations de physique du globe impose la prospection de sites parfaitement définis. En chaque site une station de mesure sera installée. Un laboratoire souterrain, section «Physique du globe» est done constitué de petites cellules, dont le montage est déterminé par la présence d'un phénomène donné et qui seront déplaçées au bout de laps de temps plus ou moins longs. L'emplaçement des cellules ne se limite pas à une seule grotte, au contraire, et il faut même envisager la pose de stations en dessous de la surface aquifère, accessibles uniquement en scaphandre, ou bien de stations automatiques reliées par fil à un enregistreur en position centrale.

b - Infrastructure - Le fonctionnement d'un laboratoire souterrain de biologie ne nécessite pas une installation exagérement compliquée: des tables et des bacs, quelques canalisations (électricité, eau, éventuellement air comprimé), éclairage. Dans le cas de la physique du globe, un matériel sommaire sera suffisant dans certains cas: une planche, un limnigraphe et quelques matériaux légers permettent d'installer une station hydrologique. Mais d'autres recherches imposent l'utilisation de moyens importants: sources gazeuses, courant stabilisé, etc... La mécanique des roches suppose l'emploi d'une sondeuse légère, donc la pose de rails et l'emploi de wagonnets.

Resumons ce paragraphe consacré aux laboratoires souterrains en soulignant qu'il s'agit d'un domaine aux multiples possibilités à peine recensées.

\section{$2^{\circ}-$ Expérimentation «in vitro»}

En se reportant à la fig. 3 nous voyons que les techniques de mesures de la physique du globe (diagonale fluides-géochimie) nous apportent un certain nombre de chiffres dont l'interprétation dans le cadre du phénomène karstique s'effectue en fonction de critères géodynamiques, à l'échelle du massif.

L'élaboration d'une théorie générale suppose la référence à des lois générales guidant l'interprétation des faits observés ou mesurés, mais l'insertion dans un complexe de facteurs interférant, ne rend pas évidente l'application de ces lois générales, et il devient alors nécessaires de procéder à certains contrôles de validités.

Ces contrôles pourront être de nature variable suivant le degré d'avancement de la recherche. Il pourra s'agir de la simple vérification 
d'une hypothèse très générale suscitée par une observation de terrain. Une expérience simple (expérience démonstration) pourra montrer que le mécanisme envisagé est possible ou non. C'est ainsi que Melle Pobeguin a prouvé que les concrétions d'aragonite résultent de l'alimentation par une eau sursaturée en bicarbonate de calcium.

Des investigations plus fouillées imposent la mise en œuvre de moyens importants; des mesures systématiques permettront d'élaborer une théorie de la sédimentation limono-argileuse en conduit karstique. A partir d'échantillons recueillis en caverne, de granulométrie et minéralogie connue par l'emploi de méthodes de mesures classiques (pipette de Robinson, liqueurs denses), il est possible d'appliquer les lois «de la rhéologie et de la physico-chimie des suspensions» à ce matériel, les variations propres au milieu, le conduit karstique en l'occurence, étant contrôlées «in vitro». En France, l'étude de la sédimentation argileuse en caverne a été ainsi esquissée par J. J. Blanc.

Enfin lorsque le problème atteint un niveau donné de complexité il devient nécessaire d'envisager, tout au moins de programmer, un modèle réduit. D'usage courant en hydraulique fluviale, il semble que cette technique n'a pratiquement pas été utilisèe pour résoudre des problèmes d'hydrologie karstique.

Ces principaux résultats, physiques ou physico-chimiques, étant connus il sera possible de les utiliser d'un point de vue plus général pour reconstituer l'évolution d'un karst. Mais nous quittons alors le domaine expérimental pour aborder la reconstitution paléogéographique impliquant des formes de raisonnement différentes.

\section{La corrosion expérimentale}

A titre d'exemple nous allons présenter un schéma des recherches expérimentales effectuées lors d'études portant sur les diverses modalités d'attaque chimique d'une roche soluble. Deux étapes peuvent ètre envisagées, reposant sur des principes assez différents.

Il est d'abord indispensable, au cours d'une investigation rapide, de classer les modalités d'attaque karstique d'une roche soluble. Dans la nature, le cas le plus général est constitué par l'action d'une eau chargée de $\mathrm{CO}_{2}$ agissant sur le carbonate de calcium. Et alors, la corrosion ne se limite pas à une simple dissolution de la roche. La décomposition du carbonate reposant sur l'action d'un acide faible, est constituée par une forte hydrolyse entrainant de complexes variations d'équilibres soumises aux facteurs naturels. Il s'agit d'un phénomène lent, nécessitant pour une reproduction au laboratoire un 
dispositif expérimental important. Afin de débrouiller le problème, au cours d'une première phase nous nous contenterons de soumettre le carbonate à l'action d'un acide fort, ceci afin d'identifier les mécanismes de détail intervenant dans la corrosion. Les «attaques d'acides» constitueront la première partie de ce chapitre.

Ensuite nous évoquerons les processus naturels en citant les travaux conçernant les équilibres $\mathrm{CO}_{2}-\mathrm{H}_{2} \mathrm{O}-\mathrm{CO}_{3} \mathrm{Ca}$ et les éléments qui les déterminent. Dans cette $2^{\circ}$ partie nous considérerons donc les possibilités de fonctionnement des mécanismes envisagés dans la première partie. Nous serons beaucoup plus bref, l'établissement d'une théorie de la corrosion reposant beaucoup plus sur les investigations de terrain que sur les mesures de laboratoire.

\section{$1^{\circ}$ - Attaques à l'acide}

\section{A-Mesures}

L'analyse chimique d'un roche carbonatée repose sur la mise en solution de la roche dans un acide suivie du dosage des cations, par exemple en utilisant la méthode du versénate (Vatan).

Cette analyse chimique peut s'effectuer sous une forme simplifiée. Mentionnons la réaction qualitative au jaune thiazol pour déceler le magnesium, la dolomimétrie en utilisant une méthode conductimétrique après attaque à l'acide sulfurique (Fouquet, Capdecomme) ou la calcimétrie au densimètre (Saporte).

Rappelons les calcimétries par mesure du volume de $\mathrm{CO}_{2}$ dégagé à la suite d'une attaque à l'acide chlorhydrique dans le calcimètre modèle Bernard, très répandu, ou bien dans le calcimètre Dietrich Fruhling plus précis mais d'emploi compliqué.

En minéralogie il est courant d'identifier certains minéraux par coloration sélective. Pour distinguer la calcite de la dolomie, faciles à confondre en lame mince, l'on fait agir sur une surface polie une solution d'un sel métallique qui attaque faiblement la calcite et non la dolomie, puis à colorer par une réaction secondaire le composé plus ou moins complexe qui s'est formé sur la calcite. Ce sont les colorations au chlorure ferrique, ou bien au nitrate d'argent.

Ces colorations peuvent intervenir après attaque à l'acide d'une surface polie suivant la méthode de Lamar, consistant à soumettre l'échantillon, soigneusement poli, à une attaque de $20 \mathrm{mn}$ à l'acide acétique (23 cc pour 100 cc $\mathrm{H}_{2} \mathrm{O}$ ) ou de $5 \mathrm{mn}$ à l'acide chlorhydrique $\mathrm{N}$ (8 cc pour $100 \mathrm{cc} \mathrm{H}_{2} \mathrm{O}$ ). Ces deux attaques donnent des résultats différents et complémentaires. Avec l'acide chlorhydrique, la faible solubilité différentielle dégage la structure en mettant bien la dolomie en 
relief, alors que l'acide acétique, avec une forte solubilité différentielle, qui est à rapprocher de l'action du $\mathrm{CO}_{2}$ dans la nature (cette comparaison étant proposée sous toutes réserves), souligne tous les détails intracristallins et donne des surfaces d'attaque proches de ce que l'on peut observer dans la nature.

Recemment une publication russe (Kudrin) a proposé une méthode rapide permettant de classer les roches carbonatées suivant leur grain, teneur en dolomite et en impuretées, en les soumettant à l'attaque de solution à $0,5,1,2,5,8,10,15,30 \%$ et concentrées de ClH la roche faisant effervescence ou restant intact pour une concentration donnée. En outre l'auteur recommande de faire les essais successivement sur l'échantillon en fragment, la réaction étant alors contrôlée par la teneur plus ou moins grande en impuretées, ou bien la roche étant réduite en poudre, la réaction devenant alors très sensible à la teneur en carbonate de calcium.

L'analyse des résidus insolubles constitue une technique pétrographique d'usage courant chez les sédimentologistes. Les résidus subsistant, sable ou argile, permettent une analyse granulométrique, voir une extraction des minéraux lourds.

L'étude de la genèse biochimique de certains calcaires utilise également l'attaque d'acide (méthode Tixier-Durivault, cit. Nesteroff). Après attaque à l'acide acétique $1 \%$ additionné de bleu de méthylène le substratum organique apparait sous la forme d'une masse gélatineuse colorée, ou bien sous forme de trame organique. Nous renvoyons aux publications correspondantes, tout en rappelant que c'est une technique analogue, qui, après attaque, montrant la présence de résidus organique dans un mondmilch (Hoeg, cit. Davies et Moore) a conduit à admettre l'origine bactérienne de ce produit.

Les attaques à l'acide sont couramment utilisées en technique paléontologiques pour isoler les fossiles inclus dans une roche, lorsque les fossiles sont formés de matériaux insolubles, ou pour dégager les coquilles plus résistantes par solubilité différentielle (acide lactique très dilué par exemple).

Enfin les spécialistes de la porométrie ont annexé le procédé. La roche est imprégnée sous vide d'une résine synthétique colorée par ex. de l'Araldite, indurant la roche, ce qui facilite la taille de P.M. et permettant par examen au microscope de caractériser la porosité de l'échantillon (Etienne). La roche étant ultérieurement attaquée à l'acide, subsiste alors un moulage à trois dimensions des pores de la roche.

L'industrie a même été touché par cette technique. Il est devenu courant d'«acidifier» un sondage pour augmenter la section des 
canalicules d'alimentation et favoriser l'alimentation en eau d'un captage.

\section{B - Expériences «au filet d'eau acidulé»}

Les premières expériences assimilant un causse à une dalle calcaire («Les causses artificiels» - Meunier, 1894) et les eaux naturelles agressives à un «goutte à goutte» acidulé semblent dues à Stanislas Meunier.

Une dalle calcaire ayant été brisée à la masse en plusieurs fragments ensuite rapprochés, l'on fait arriver le liquide corrosif en contact avec une fissure. Celui-ci s'infiltre dans le vide de la fêlure, ne tarde pas à l'élargir et bientôt on constate qu'il s'y développe des effets d'une grande ressemblance avec les phénomènes naturels «Les filets corrosifs tendent à se concentrer le long de certaines verticales, pendant que les régions voisines sont laissées intactes». "Ils arrivent ainsi à creuser des séries d'avens alignés sur une fine fissure dont ils constituent comme des élargissements locaux.»

Nous avons reproduit cette expérience au laboratoire de Moulis en utilisant un bloc de calcaire crétacé de la région de Montpellier, épais d'environ $7 \mathrm{~cm}$, divisé au marteau en trois fragments. Nous avons fait suinter un "goutte à goutte» d'eau acidulée ClH d'environ N/3. Les conditions d'impact sur un croisement de fêlures d'où un éclat avait sauté créant une pseudo-doline d'origine mécanique faisaient de cette expérience un cas particulier. Le bloc reposait sur la faïence d'un évier et le liquide agressif ayant traversé le bloc ruisselait sur ce socle insoluble, baignant la base du bloc avant de gagner l'orifice de vidange (fig. 4).

La forme résultante, liée probablement à la lenteur du débit, est caractéristique d'une certaine modalité d'attaque. L'orifice supérieur est demeuré d'une section à peine plus grande que la cavité faisant suite. Les gouttes rebondissaient sur le rebord du bloc A qui a été partiellement attaqué et venaient ruisseler sur l'autre face, opposée, y façonnant une dépression peu profonde $(4 \mathrm{~mm})$, mais relativement large $(3 \mathrm{~cm})$, divisée verticalement en deux zones. La partie supérieure se caractérise par une surface lisse sauf en bordure où la "paroi» est soulignée par une guirlande de petits chicots allongés dans le sens. apparent des filets liquides. Vers le bas l'alvéole supérieure se transforme en petits canaux subverticaux, en éventail se réunissant vers le bas en deux collecteurs, séparés par une arête aigüe ou plane correspondant à l'ancienne adhérence avec l'autre face de la cassure L'exutoire inférieur est constitué par une petite fente de $3 \mathrm{~cm}$ de long, 


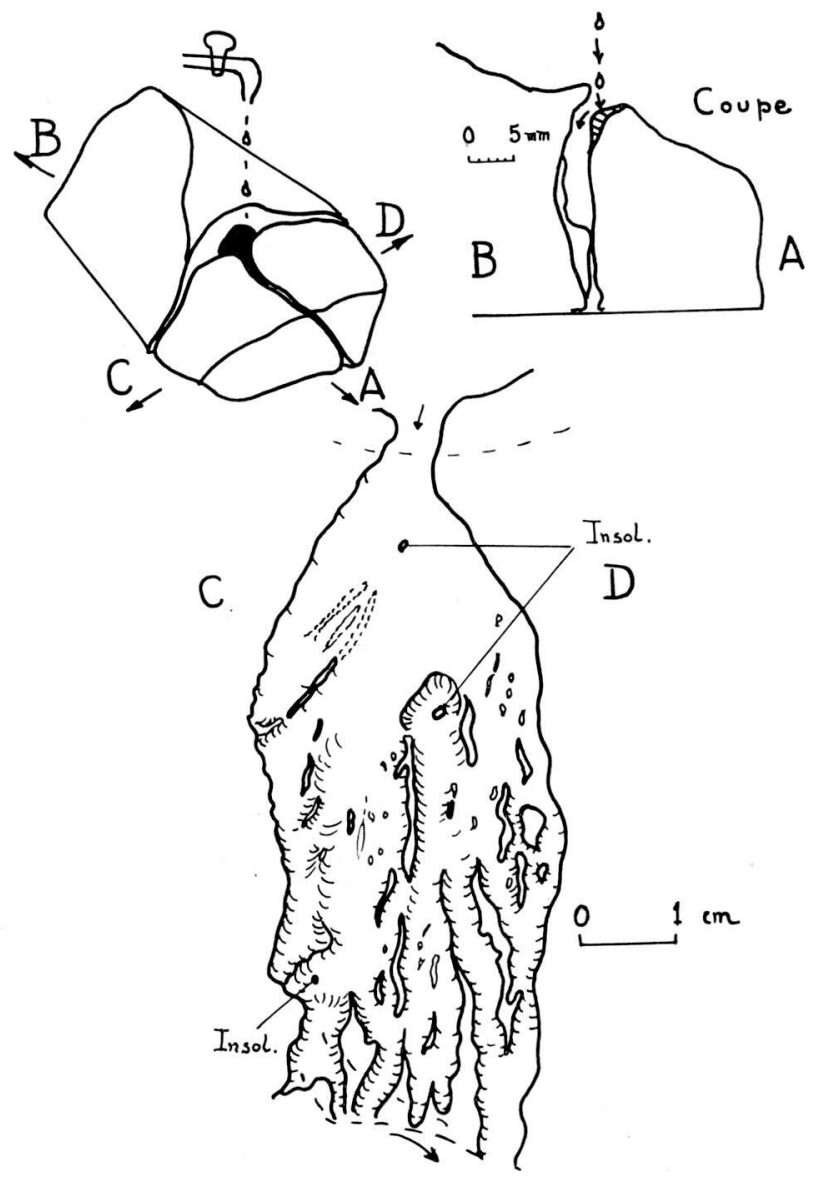

Fig. 4: Expérience de "Causse artificiel». - En haut à droite: vue générale du bloc attaqué, à gauche: coupe. - En bas: agrandissement de la face B de la fissure.

$1 \mathrm{~mm}$ de large s'élargissant vers le centre en canalicule subciculaire de $4 \mathrm{~mm}$ de diamètre, limitée par des arêtes aiguës vers le bas.

Dans ce façonnement il faut noter l'importance des éléments insolubles ou peu solubles inclus dans la roche. Dans la partie supérieure de l'alvéole, un minuscule filonnet de matière jaune occupant le centre de la surface plane a constitué un centre de dispersion des filets liquides, marqué par la disposition en éventail des arêtes subsistantes. Un peu plus bas un autre filonnet insoluble a également servi de centre de dispersion d'où la disposition en éventail des canalicules de la zone 
inférieure, mais en outre, autour de cet élément moins soluble s'est façonné une cupule de $7 \mathrm{~mm}$ de diamètre, s'enfonçant de $4 \mathrm{~mm}$. Enfin à la partie inférieur du bloc l'un des petits collecteurs se superpose à un microfilonnet de calcite.

Si nous avons insisté sur cette expérience c'est qu'elle réalise un programme relativement proche d'une certaine réalité et que les formes obtenues présentent des homologues dans la nature.

Il n'en est pas ainsi de toutes les expériences réalisées par St.Meunier. Faisant aboutir un filet d'eau acidulé sur bloc calcaire non fissuré, il observe la formation d'une cavité au point d'arrivée du liquide. A la longue cette cavité réalise la perforation verticale du bloc et à partir de ce moment «c'est vers le bas que se porte le maximum d'action dissolvante et le fond tend à s'élargir». Un éteignoir s'esquisse vers le bas. La cavité prend une forme en sablier et, finalement, la perforation devient un tronc de cône s'élargissant vers le bas.

De la même façon un petit jet vers le haut provoque un creusement en éteignoir remontant, ainsi qu'il était facile de le prévoir.

L'auteur attire l'attention sur cette forme en éteignoir et la met en rapport avec l'allure fusiforme de la plupart des puits. Or à quoi est dû la forme en entonnoir renversé de la perforation d'une dalle calcaire sous l'action d'un filet? Simplement au rebondissement des gouttelettes sur le support du bloc, alors qu'il semble bien que la genèse des puits naturels résulte d'un suintement le long des parois. L'écart est trop grand entre la réalité et le montage expérimental.

Nous avons cherché à reproduire cette expérience, à titre d'information, et constaté que la formation d'un entonnoir à la partie supérieur d'un bloc ne se produit que lorsqu'un certain nombre de conditions favorables se trouvent réunies. La plupart du temps un filet de liquide agressif s'écoule à la surface du bloc, corrodant un chenal sur son parcours. Pour un certain débit (lent) un dispositif de type microfluviatile est progressivement façonné. Prenons un exemple (fig. 5): le bloc soumis à attaque est formé de calcaire urgonien recristallisé provenant des carrières de Couflens (Ariège). La surface soumise au ruissellement est perpendiculaire au litage de la roche, c'est à dire qu'une attaque à l'acide met en évidence l'existence de lits bien cristallisés qui demeurent en relief au milieu d'une pâte microcristalline moins résistante. Au point d'impact des gouttes un certain nombre de lames ( $1 / 10 \mathrm{~mm}$ d'épaisseur) bien cristallisées, progressivement isolées, apparaissent dans un périmètre donné, un petit goulot de drainage s'établissant au point le plus bas. Lorsque l'épaisseur de la zone attaquée au point d'impact devient suffisamment importante le choc des gouttes brise les lamelles de calitce et au point d'arrivé du filet 

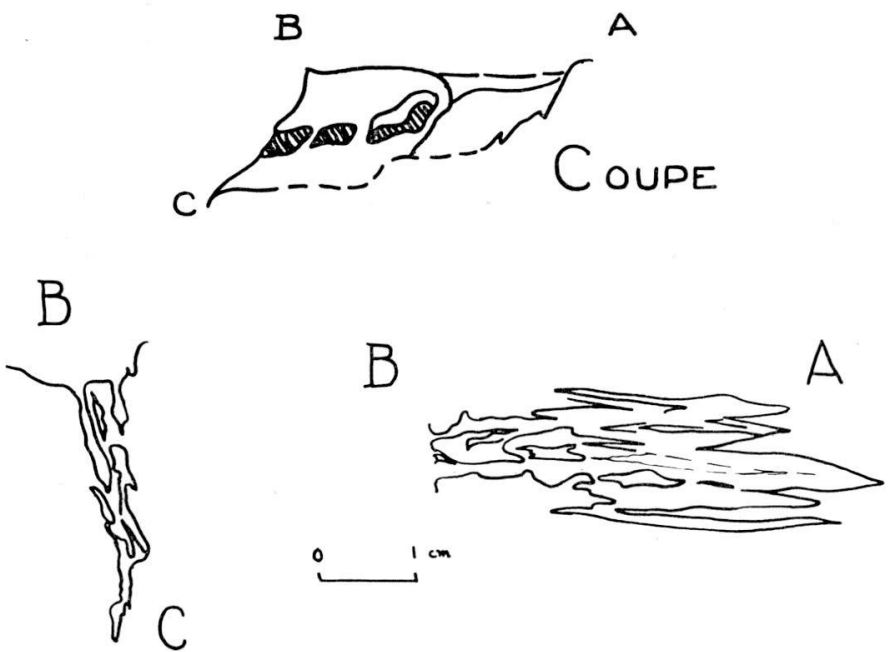

Fig. 5: Expérience d'attaque d'acide: influence de la solubilité différentielle.

liquide apparait un entonnoir de forme irrégulière, allongé dans le sens du litage. Le goulot de drainage évolue par incision d'une zone «tendre» entre deux lamelles de calcite, la fente résultante ayant une ouverture de $1 / 2$ à $1 \mathrm{~mm}$ et s'approfondissant progressivement. En profondeur $(1 \mathrm{~cm})$ un relais s'établit d'une zone tendre à une autre zone tendre parallèle à la première, c'est à dire que l'écoulement initialement "aérien", mais en étroit canyon, devient "souterrain", c'est à dire s'effectue sinon en «conduite forçée» tout au moins, en fonction de l'alimentation goutte à goutte, en régime de «crue suivie d'un drainage rapide». Rappelons à cette occasion que le canyon peut être considéré comme une "grotte à ciel ouvert». Cette remarque s'applique spécialement à l'expérience ici mentionnée.

Citons pour mémoire le jet oblique aboutissant sur une fissure et façonnant une galerie modèle réduit.

D'une façon générale ces «expériences démonstrations» furent utiles en 1900, alors que les controverses portant sur la genèse des cavernes les théories de l'origine profonde (séisme ou conduit volcanique) ou synsédimentaire (dégagement de $\mathrm{CO}_{2}$ avant consolidation des couches) s'opposaient encore à la conception hydrologique - étaient encore présentes à toutes les mémoires et qu'il importait d'asseoir la théorie attribuant leur véritable rôle aux fissures et à la corrosion des eaux acidulées. Pédagogiquement elles demeurent utiles pour illustrer un cours d'initiation à la karstologie, présenté par un moniteur discutant la valeur démonstrative du montage réalisé. 


\section{C-Expériences de corrosion}

Ces expériences de "Causse artificiel» envisagent directement la reproduction à échelle réduite du phénomène karst tel qu'il est globalement observé par le spéléologue. Moins ambitieux d'autres chercheurs ont voulu préciser «in vitro» le mécanisme de la corrosion géochimique. Parmi ceux ci il convient de mentionner spécialement G. Cosyns, alors (1909) assistant au Laboratoire de Chimie de l'Université libre de Bruxelles, et qui a joué un rôle d'initiateur en ce domaine.

De nombreuses expériences peuvent être envisagées. Nous grouperons en cinq rubriques les diverses tentatives venues à notre connaissance.

a - Rôle respectif de l'érosion et de la corrosion:

En 1908, G. Cosyns étudie la perte de poids de plaquettes calcaires disposées sous un filet liquide: d'abord d'eau pure, puis d'eau acidulée, d'eau chargée de sable, d'eau acidulée chargée de sable. Il conclut à la prépondérance de la corrosion dans l'attaque du calcaire (Van den Broeck).

Par contre en 1953, P.Chevalier, avec un protocole opératoire différent, démontre exactement l'inverse. Les échantillons sont fixés sur une grille inoxydable pour éviter les frottements entre blocs. Les grilles sont disposées dans un flacon contenant de l'eau et du sable et mis à tourner pendant 15 jours. La perte de poids obtenue est comparée avec celle d'échantillons de même nature laissée dans l'eau pendant un mois dans un flacon bouché sous atmosphère riche en $\mathrm{CO}_{2}$, périodiquement renouvellée. La perte due au frottement sableux est nettement plus importante.

Ces expériences ne prouvent rien par elles même, mais la contradiction de ces deux résultats est intéressante du simple fait qu'elle montre une réalité plus complexe que le phénomène envisagé par les expérimentateurs.

b - Influence de la structure du calcaire:

La résistance des calcaires à la corrosion en fonction de leur composition minéralogique et de leur structure est un sujet abordé très anciennement. En 1883 Kendall étudiait les différences de résistance chimique des coquilles fossiles suivant leur composition en calcite ou en aragonite, ceci en les plongeant dans une eau chargée de $\mathrm{CO}_{2}$ (voir aussi Cornish et Kendall).

Vers 1909 G. Cosyns étudie, en liaison avec les travaux de van den Broeck, la "production résiduaire» de certains calcaires. Alors qu'un calcaire viséen lui donne $3 \%$ d'insolubles (Viséen sup.), un calcaire 
crinoïdique tournaisien laisse un important résidu. En soumettant un fragment de ce calcaire à l'action d'un acide chlorhydrique fortement étendu, il met en évidence une dissolution en 3 phases successives:

1. Au cours de la première on assiste à une dissolution rapide de la pâte formée de petits cristaux de calcite enrobés et pénétrés de petites mouches argileuses et charbonneuses. Ces impuretées forment un véritable réseau plus perméable que la calcite «et qui joue vis à vis du dissolvant, en quelque sorte, le rôle d'une mèche».

2. Subsiste alors un important résidu formé de rhomboèdres de dolomie qui se dissolvent assez lentement, laissant subsister

3. des articles de crinoïdes, des polypiers, etc... qui «ont résisté pendant plus de $24 \mathrm{~h}$ à l'acide chlorhydrique étendu» mais qui, pulvérisés et soumis à chaud à l'acide un peu plus concentré finissent par se dissoudre en abandonnant une petite quantité de $\mathrm{SiO}_{2}(1 \%$ de la masse des fossiles). L'auteur explique par la présence de cette silice l'exceptionelle résistance à la corrosion de cette fraction de la roche.

4. Reste un résidu insoluble d'argile, d'oxyde de fer, de quartz.

Ces résultats expérimentaux ont été comparés au remplissage du réseau de fentes. Les expériences avaient d'ailleurs pour but de préciser les caractères filtrants des conduits karstiques des calcaires tournaisiens.

En dehors de ce point de vue pratique, ces expériences ont eu le grand interêt de montrer le rôle des insolubles dans les mécanismes d'altération des calcaires. Un calcaire viséen soumis à l'action d'une solution $\mathrm{ClH} 1 \%$ perd $70 \%$ de sa masse en $24 \mathrm{~h}$. A partir de ce moment les fragments subsistants enrobés dans le résidu argileux constituent une masse très résistante à la corrosion et l'obtention des $9 \%$ de résidus réellement insolubles de la roche demande un temps très long. La même observation a été faite en dissolvant un autre échantillon de «calcaire argileux givétien».

Il s'agit là d'expériences, à mon sens très importantes, et que nous projetons de reprendre sous forme systématique en étudiant divers types de roches pétrographiquement bien définies et en mesurant la vitesse d'attaque la roche étant disposée sur le plateau d'une balance enregistreuse.

c - Influence de la température:

L'influence de la température sur la vitesse et l'ampleur des karstifications a fait l'objet de nombreuses publications et de controverses variées. P. Birot a fait mention sommaire d'«expériences faites en 1957 à l'Institut de Géographie de Paris et qui ont montré que c'est seule- 
ment lorsque la durée d'imbibition dépasse 3 jours que la teneur des eaux froides $\left(5^{\circ}\right)$ en calcaire l'emporte sur celle des eaux chaudes $\left(25^{\circ}\right)$ ".

d - Influence de la vitesse d'écoulement de l'eau agressive:

La vitesse d'écoulement a été considérée par certains auteurs comme un argument permettant de nier ou de limiter de rôle de la corrosion dans la spéléogenèse. Suivant un mode de raisonnement anthopomorphique l'écoulement serait trop rapide pour que la dissolution ait le temps de s'effectuer: par suite de la vitesse «les élements d'affinités n'auraient pas le temps de rester en présence».

Pour critiquer cette conception nous avons utilisé une image relevant de l'expérience commune «La vitesse de l'écoulement ne supprime pas plus la formation de bicarbonate soluble que l'agitation d'un morceau de sucre dans une tasse n'empêche sa mise en solution. (Renault, 1960».

En 1957 C. A. Kaye a publié le compte rendu de quelques expériences montrant que la vitesse de circulation ou l'agitation du solvant au contact de la surface calcaire détermine la vitesse d'attaque (fig. 6). Dans la première expérience un petit bloc est partiellement immergé dans une solution de $\mathrm{ClH}$ dilué. Autour du bloc la ligne correspondant au niveau du liquide est soulignée par une rainure. En ce point en
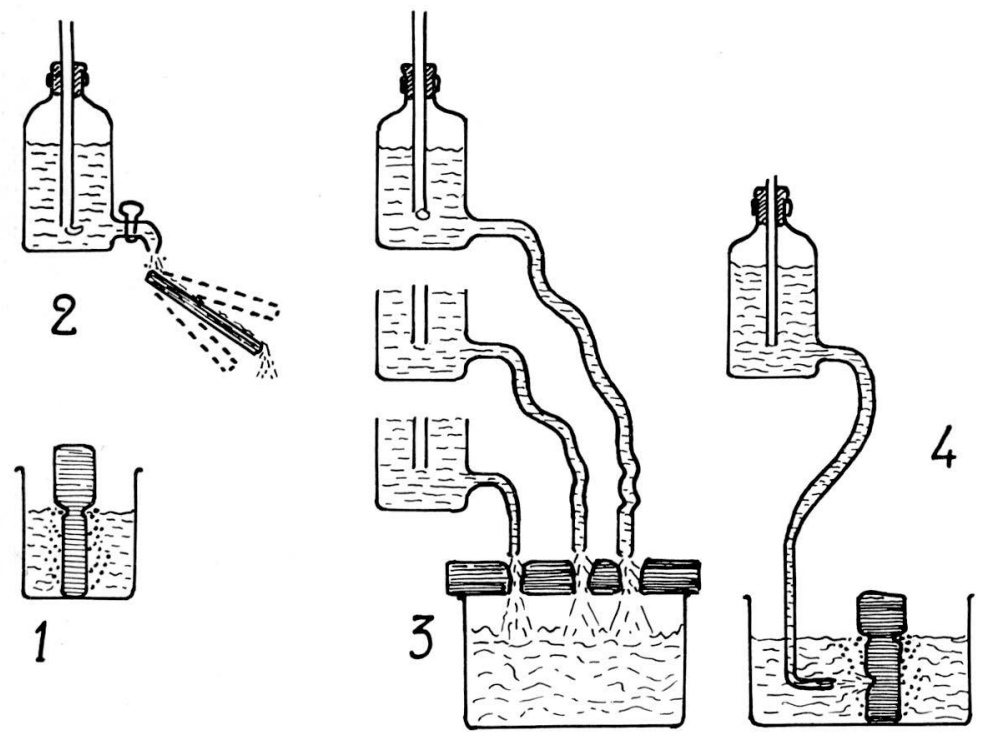

Fig. 6: Influence de la vitesse d'écoulement sur la corrosion (expériences de Kayes). 
effet les bulles d'anhydride carbonique venant crever en surface provoquent une agitation qui accélère l'attaque du calcaire. La deuxième expérience montre que l'acide ruisselant sur une dalle calcaire attaque proportionnellement à l'inclinaison de la dalle. La troisième expérience repète la même opération la circulation s'effectuant cette fois dans les perforations traversant une dalle. Enfin, pour la quatrième expérience, un tube de verre projette un jet d'acide en un point particulier d'un bloc immergé et détermine en ce point la formation d'une alvéole.

L'auteur étend le résultat de ces expériences aux phénomènes karstique et met en rapport les zones de circulation rapide et de corrosion maximum. Ce qui est logique, en l'attente de recherches complémentaires envisageant la combinaison des actions chimiques et mécaniques. Ce qui est moins logique par contre c'est de voir un auteur connu pousser la théorie à ses plus extrêmes conclusions et déclarer que les marmites de géant sont un phénomène de corrosion pure $^{5}$ ) en appuyant cette hypothèse (non suivie de vérification) sur une affirmation manifestement fausse: la microforme marmite ne s'observerait pas dans les roches non solubles ${ }^{6}$ ).

e - Réalisations micromorphologiques expérimentales:

Sans vouloir réaliser tout un Karst dans un bocal, il serait malgré tout intéressant d'obtenir artificiellement certaines microformes au laboratoire. Van den Broeck et al. (p. 1591) nous dit: «Cosyns a pu reproduire absolument tous les aspects rencontrés dans les grottes les plus tourmentées: cupulation," etc... Malheureusement il ne fournit aucun détail de réalisation ni aucun exemple de résultat obtenu. Tout au plus est il possible de citer une photo de ce que l'auteur baptise «conjonction cupulaire», coalescence de cupules aboutissant à de fines rainures correspondant probablement à un détail structural de la roche.

Le même Cosyns a constaté que la corrosion était plus rapide sur parois verticales que sur les éléments de surfaces horizontales. Dans ce dernier cas les résidus de dissolution accumulés ralentissent l'attaque de la roche.

Cette influence de la pente sur la micromorphologie a été précisée dans les expériences de Smith et Albritton, appliquées par Hoffmeister

5) Cosyns était arrivé à la même conclusion, d'ailleurs sans être suivi par Van den Broeck et al. qui rapportent cette opinion (p. 1590).

6) Il suffit de mentionner le Jardin des glaciers de Lucerne, constituant peut être un record mondial au point de vue dimensions pour les marmites, et qui est installé dans un grès quartzeux insoluble (A. Heim). 
et Ladd à un problème morphologique développé à l'échelle du paysage. Ces expériences ont montré que les surfaces inclinées de moins de $3^{\circ}$ sont piquetées de petites dépressions assimilées aux tinadjitas (et comparables aux entonnoirs des causses artificiels de St.Meunier), sur les pentes de 3 à $6^{\circ}$ se développent des facettes de dissolution et au-dessus de $20^{\circ}$ apparaissent des cannelures parallèles analogues à celles que nous décrivons plus haut dans notre creusement de micro aven.

Citons également le travail récent de Mowat.

\section{D - Expérience de corrosion à travers un remplissage}

Il est admis, bien que certaines controverses tendent à s'esquisser à ce sujet, qu'une roche calcaire peut être corrodée à l'air libre lorsque certaines conditions se trouvent remplies (Renault, 1950), mais également sous remplissage, le régime d'attaque étant lié à la perméabilité et à la possibilité d'échanges gazeux du remplissage ${ }^{7}$ ).

Mentionnons pour être complet une expérience de St. Meunier ayant pour but de démontrer l'existence de ce que cet auteur baptisait «sédimentation souterraine» en donnant à cette expression un sens très particulier qui n'a pas été retenu en géologie. Pour Meunier il y aurait sédimentation souterraine lorsqu'un banc formé de roche soluble, contenant des éléments insolubles est traversé par des infiltrations agressives, la partie soluble du banc disparait progressivement, les éléments insolubles subsistant seuls. Il explique ainsi les sables phosphatés de Picardie, les bone-beds de l'Infra-lias anglais, etc...

Pour illustrer cette interprétation l'auteur avec un tampon d'amiante obture la base d'un tube de verre et dispose au-dessus de ce tampon des lits successifs formé par un mélange de $\mathrm{CO}_{3} \mathrm{Ca}$ et de fer tamisé, ou de $\mathrm{CO}_{3} \mathrm{Ca}$ et de quartz, etc... et fait filtrer à travers cette colonne une eau acidulée au $\mathrm{ClH}$. Un tassement général en résulte, des lits de fer, de quartz, etc... apparaissant à l'intérieur du tube, et ceci à partir du haut.

Personnellement, et orientant les recherches dans une autre direction, nous avons entrepris une série d'expérience ayant pour but de préciser l'influence de la nature du colmatage sur les microformes apparaissant sur les blocs noyés dans un dépôt perméable, ou bien sur les surfaces rocheuses recouvertes par les mêmes dépôts.

Il est en effet admis depuis les travaux de Chaix, Du Bois, Martel, Van den Broeck, etc... que les cupules de la corrosion alvéolaire des

7) Nous ne tiendrons pas compte ici de la genèse des sols pédologiques où interviennent des processus biologiques hors de notre sujet. 
parois de grottes se forment en régime noyé ou libre, mais en conduit souterrain et sur paroi vierge de tout sédiment ${ }^{8}$ ), alors que, sous remplissage les formes sont arrondies, sans arêtes aigües ni lames coupantes.

En outre dans une publication antérieure nous avons posé le problème de la genèse des vagues d'érosion (Renault, 1961) sous remplissage et il était nécessaire de tenter un contrôle expérimental. Sans apporter de réponse définitive, cet essai fournit des informations complémentaires (Renault, 1963).

Nous avons réalisé le montage suivant (fig. 7):

- l'alimentation est réalisée goutte à goutte par un vase de Mariotte remplie d'une solution $\mathrm{ClH}$ de 1 à $5 \%$; lorsque un débit important s'impose, le vase de Mariotte est remplaçé par une jarre de 5 litres, un robinet permettant de régler le débit d'eau acidulée,

- la cellule expérimentale est constituée par un tube de verre de $25 \mathrm{~mm}$ de diamètre, obstrué à sa partie inférieure par un tampon de laine de verre, ou bien par un grillage lorsque l'expérience réclame un débit élevé. Un petit bloc de roche soluble (calcaire, dolomie ou autre) est disposé sur le tampon à l'air libre ou bien noyé dans un produit meuble dont les caractéristiques sont connus,

- dans le cas où le protocole de l'expérience réclame une percolation lente, le tube est immergé dans un bècher, le bord supérieur du tube dépassant de quelques mm seulement le niveau du bec du bécher.

La première expérience (fig. 7), jouant un rôle témoin, avait pour but d'examiner les effets d'une circulation acidulée lente sur bloc immergé. Comme prévu le passage de 500 cc de solution acidulée a pour effet de mettre en relief les détails structuraux tels que les montrent une surface polie soumise à une attaque d'acide suivant les normes établies par Lamar.

Dans la $2^{\circ}$ expérience le bloc, noyé dans un produit fin (sable de Fontainebleau Prolabo lavé aux acides, diamètre médiane $0,185 \mathrm{~mm}$, sigma $=0,8$ alpha) était baigné par la même solution percolant à travers le sable. Après interruption et extraction du bloc, celui-ci présentait trois types de surfaces différentes:

a-sur une facette s'observait encore les stries du découpage initial à la scie diamantée,

$\left.{ }^{8}\right)$ Les alvéoles se formant en lapiaz, ou sur falaise, dans la zone extérieure au milieu souterrain, c'est à dire soumise aux variations de température et d'hygrométrie, aux actions biologiques, etc... sont beaucoup plus proches des cupules façonnées en régime enterré, que les cupules de cavités non colmatées. 


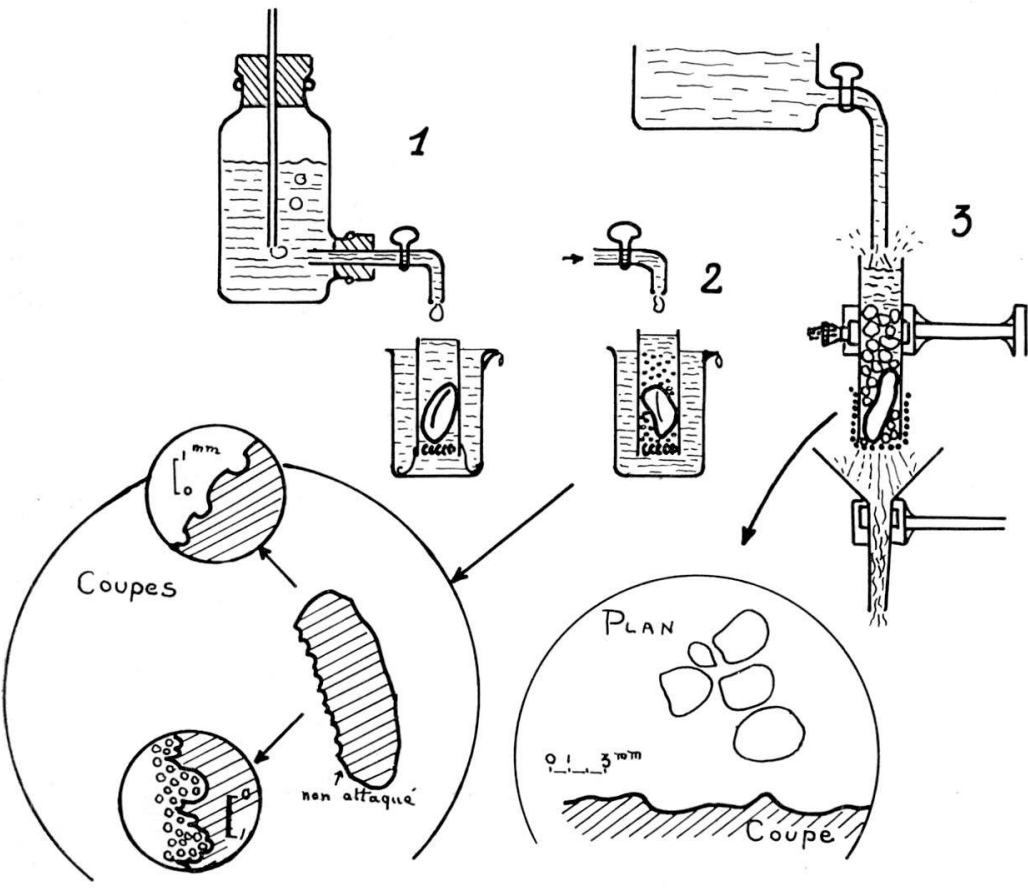

Fig. 7: Expériences de corrosion sous remplissage.

b - à la partie supérieure du bloc et latéralement, la surface était arrondie et entaillée par des cannelures vermiculées orientées très approximativement suivant la pente, larges de 0,3 à $0,5 \mathrm{~mm}$ et longues de 4 à $10 \mathrm{~mm}$,

c-deux faces protégées par un surplomb étaient couvertes de cupules coalescentes séparées par des arêtes anguleuses, aucune orientation préférentielle n'apparaissant dans les alignements, la profondeur des cupules étant proportionnelles à leur diamètre. Au voisinage des arêtes s'observaient de petites cupules élementaires de 0,2 à $0,4 \mathrm{~mm}$ de diamètre, arêtes et cupules élémentaires entourant des alvéoles plus grandes, de 0,5 à $1 \mathrm{~mm}$ de diamètre, aux parois lisses ou entaillées de cupules secondaires peu profondes, ou avec détails structuraux en relief comme dans le cas d'une attaque sans percolation.

Cette $2^{\circ}$ expérience peut être interprétée comme suit. Les faces supérieures bénéficiaient du rôle protecteur de l'accumulation sableuse. L'expérience ayant débuté par un essai de perméabilité à l'eau distillée, un isolement par agrégats maintenus par l'eau capillaire peut être envisagée. Au fur et à mesure de l'attaque les grains de sable en se 
déplaçant, répartissent la corrosion sur toute la surface du bloc. Un certain nombre de filets liquides, suivant une voie intersticielle entre grains et parois, burinent les petites cannelures.

Les faces latérales, légèrement surplombantes, se trouvent en contact avec les grains, mais l'adhérence est beaucoup moins stricte que dans le cas précédent. Les grains orientent l'attaque, mais sans limiter la circulation du liquide acide. Le recul de la paroi, relativement rapide, aboutit, par recoupement de facettes, à la genèse de crêtes anguleuses.

Pour la $3^{\circ}$ expérience, nous avons noyé le bloc dans un remplissage grossier, formé par le refus des tamis 33 et 34 Afnor d'un sable constitué par des grains de quartz et d'orthose (médiane 1,8 mm), permettant un débit de percolation élevé: 7 à $9 \mathrm{cc} / \mathrm{sec}$. Après passage de 30000 ec de solution acidulée, l'échantillon examiné montrait des parois burinées par deux types de microformes.

Certains éléments de surface, situés en dehors de l'écoulement principal, ou bien pour des raisons structurales, se montraient couvertes de cavités irrégulières et sans orientation définies. Le reste de la plaquette portait deux types d'alvéoles:

- de petites dépressions symétriques d'environ $1 \mathrm{~mm}$ de diamètre, pour à peine $0,1 \mathrm{~mm}$ de profondeur,

- des cavités de 2 ou $3 \mathrm{~mm}$ de diamètre, approximativement aussi longues que larges, sans être circulaires pour autant, profondes de $0,5 \mathrm{~mm}$ environ, beaucoup d'entre elles reproduisant la forme des vagues d'érosion observées en caverne, les détails structuraux de la roche intervenant pour déformer ces creux. Il faut noter que les vagues expérimentales nous ont paru infiniment plus dissymétriques que les vagues naturelles observées par nous.

Cette $3^{\circ}$ expérience montre la possibilité de ce mécanisme envisagé par nous dans une publication antérieure pour expliquer la genèse des vagues d'érosion. Un écoulement rapide s'effectuant le long d'une paroi formée de matériaux solubles, les filets d'eau sont orientés par les masses insolubles ou moins solubles, qui provoquent la formation de sillages, avec accélération sur la face amont de l'obstacle, d'où corrosion plus rapide, et ralentissement aval et donc réduction de l'attaque.

Cette dernière réalisation nous parait intéressante. De nombreux mécanismes ont été proposés pour expliquer la genèse des vagues d'érosion certains invraisemblables, d'autres admissibles (Renault, 1961). Nous avons personnellement remarqués que, dans certaines cavernes, la présence de vagues d'érosion s'associait à un remplissage grossier (galets) insoluble (granite, quartz, grès, etc...) une corrélation 
Fig. 8: Concrétions artificielles (d'après Huff).

certaine existant entre la granulométrie du remplissage et la largeur des vagues.

Renvoyant à une publication ultérieure, où les problèmes posés par la génèse des vagues d'érosion seront traités de façon détaillée, nous nous contenterons de signaler cet accord entre mesures de terrain et vérifications expérimentales.

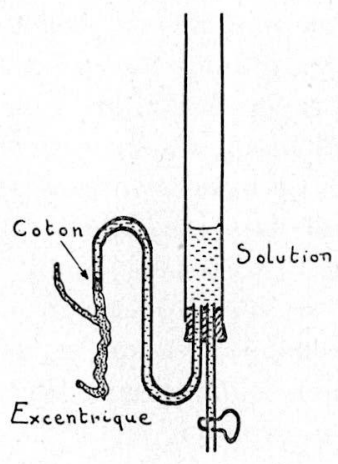

\section{$2^{\circ}$ - Concrétions artificielles}

Mentionnons rapidement les concrétions qu'il est possible de réaliser artificiellement, et rapidement, en utilisant des produits très solubles tel que $\mathrm{Na}_{2} \mathrm{~S}_{2} \mathrm{O}_{3}$ (Huff, cit. Bretz) (fig. 8) ou CINa.

Nous avons personellement observés, au cours de campagnes géologiques effectuées au Sahara (1950-54) dans les salines de Bilma (Niger) de magnifiques concrétions de $\mathrm{NaCl}$ (associé à $\mathrm{KCl}, \mathrm{Na}_{2} \mathrm{CO}_{3}$, etc...) de formation artificielle. Les saulniers récoltent le sel en creusant des cuvettes au fond desquelles l'eau saturée s'évapore. La sursaturation amène la cristallisation du sel en surface (cf. calcite flottante). Afin d'accélérer cette évaporation, les ouvriers agitent l'eau et les projections liquides sur les talus, par évaporation, donnent naissance à des concrétions de sel. Nous avons observé de minuscules excentriques, de véritables macaronis, des stalagmites en cierge, des gours.

Farrington a démontré, en utilisant du ClNa, le rôle de l'attraction capillaire dans la genèse des stalactites.

Les boulettes d'argiles, imbibées d'eau salée, et abandonnées à l'air libre, se dessèchent rapidement et se recouvrent d'un feutrage de fines aiguilles de sel, analogues aux piprakes de glace et à rapprocher de certaines cristallisations de cavernes.

Nous n'insisterons pas davantage les concrétions ainsi obtenues étant assez différentes des concrétions observées en caverne.

\section{$3^{\circ}-$ Equilibres $\mathrm{CO}_{2}-\mathrm{H}_{2} \mathrm{O}-\mathrm{CO}_{3} \mathrm{Ca}$}

Les équilibres $\mathrm{CO}_{2}-\mathrm{H}_{2} \mathrm{O}-\mathrm{CO}_{3} \mathrm{Ca}$ ont fait l'objet de nombreuses publications d'inspirations variées, les préoccupations de l'auteur pouvant être d'ordre purement chimique, conçerner une application industrielle - la lutte contre l'entartrement des conduites ou des 
chaudières - minéralogique, hydrogéologique, etc... Le grand nombre des publications aussi bien que leur valeur inégale doit inciter à la prudence lorsque l'on cherche à appliquer certaines notions aux phénomènes naturels.

Certaines confusions seraient peut être à l'origine de contradictions relevées dans la littérature. C'est ainsi que J. Corbel, considérant que le $\mathrm{CO}_{2}$ est plus soluble à froid qu'à chaud, pense que les processus de karstification étaient particulièrement intenses en région froide et que tous les grands karsts d'Europe se sont façonnés lors des phases paléoclimatiques périglaciaires du quaternaire. P. Verdeil défend une opinion opposée ${ }^{9}$ ).

Ces deux auteurs ne sont pas chimistes. Si nous consultons les spécialistes nous découvrons d'autres oppositions. Certains auteurs admettent que les eaux de résurgence sont en général sursaturées (Caro) alors que les mesures de Roques montreraient «que la totalité des résurgences (étudiées) de débit égal ou supérieur à 2 litres par seconde» est dans la zone de sous-saturation. Il y a là un problème de vocabulaire qui demanderait à être précisé.

Nous mentionnerons pour mémoire les travaux attribuant un rôle exagéré aux sulfates présents sous forme d'impureté dans les roches carbonatées (Cosyns, Kolodiajnaya, Loriaux). En dehors des observations de terrain qui contredisent cette affirmation (Renault, 1959 et 1960), nous verrons plus loin pourquoi ce facteur ne doit pas être surestimé.

L'un des acquis importants de ces dernières années, en géochimie du karst, est la découverte d'une réversibilité de la corrosion en profondeur, à l'intérieur d'un karst calcaire ou dolomitique.

Plusieurs auteurs ont en effet remarqué que les karstifications en roche très soluble, mais non carbonatées, tel que le sel gemme, le gypse sont limitées en extension. Plusieurs explications peuvent être proposées. Par exemple le gypse résulte souvent de l'altération superficielle de l'anhydrite. Cette dernière roche est peu soluble, et ceci expliquerait l'absence de circulations à grandes distances, le réseau demeurant localisé dans la zone supérieure gypseuse.

De la mème façon il est possible d'envisager une évolution très rapide des réseaux en fonction de la grande solubilité de la roche, et aussi de son absence de résistance mécanique favorisant les éboulements. Le creusement des réseaux s'accompagnerait aussi vite de leur destruction. Mais, et ceci constitue à notre avis un argument

\footnotetext{
9) Dans un texte très nuancé, H. Roques a émis des réserves sur l'importance géochimique des dissolutions en montagne.
} 
beaucoup plus déterminant, les karstification se raréfient et disparaissent lorsque la roche soluble est surmontée par un niveau étanche. Haudour et Sarrot-Reynauld notent ainsi l'absence de karstifications dans les gypses du dôme de la Mure (Isère) lorsque ceux ci se trouvent surmontés par des marnes.

Par opposition aux karstifications intenses, mais limitées du sel et du gypse, les réseaux traversant les roches carbonatées présentent des dimensions très importantes, aussi bien dans le sens vertical que horizontal (30 à $40 \mathrm{~km}$ dans le $\mathrm{S}$. E de la France). Une comparaison morphométrique entre réseaux carbonatés et salifères demanderait pour être totalement déterminante, que l'on tienne compte des structures géologiques, ce que nous ne pouvons faire ici.

Un autre élément de comparaison intervient alors: l'existence dans les roches carbonatées d'importants réseaux de conduits horizontaux installés dans une formation calcaire surmontée par une couverture imperméable. Citons le réseau de Mammoth cave, et, en France, le ruisseau souterrain d'Orchaise (Renault, 1959), de petites dimensions, mais très caractéristique. L'existence de Karsts couverts (Cvijic, 1925) par une couverture imperméable (ceux ci ne devant pas être confondus avec les karsts sous jacents [Chabot] à des terrains meubles et perméables) est propre aux roches carbonatées. Un certain nombre de géologues ignorant cette propriété des roches calcaires, il fallait insister sur ce point.

Cette opposition entre deux types de karsts peut être expliquée de la façon suivante: dans les karsts salifères le façonnement des conduits, c'est-à-dire l'attaque chimique de la roche repose sur une simple dissolution, le creusement étant limité à la zone d'action de cette dissolution, l'aire d'extension maximum du réseau ne pouvant déborder que faiblement en dehors de cette zone. Cette conception s'accorde bien avec un réseau karstique limité aux circulations proches du versant, mais s'applique difficilement aux grands réseaux profonds en roche carbonatée.

C'est pourquoi de nombreux auteurs (Steidtman, 1936) ont cherché à montrer que la corrosion - le terme étant pris comme synonyme de dissolution - était impossible dans la partie profonde d'un karst. Or les conduits spéléologiques correspondent à une réalité indubitablement constatée, et si l'on admet que les eaux de percolation sont rapidement saturées, le creusement en profondeur résulte alors de l'action mécanique des eaux courants. Cette conclusion nous entraine vers des raisonnements difficiles à conduire, le propre d'une érosion mécanique étant de progresser de l'aval vers l'amont, alors que la corrosion s'exerce de haut en bas, dans le sens d'une saturation progressive du solvant mis en contact avec le matériel soluble. 
Or, ce que nous savons de la spéléomorphologie s'accorde mal avec ces deux conceptions. D'une part l'existence de pertes pénétrables s'accorderait avec un creusement de l'amont vers l'aval, aussi bien que certains conduits de résurgences pourraient être interprétés en terme d'érosion régressive, dans le sens aval - amont. Mais d'autre part les réseaux profonds, accessibles par d'étroits passages, se développent de façon apparemment indépendante de la topographie externe. Ce creusement localisé à un niveau donné demande à être expliqué.

En 1953, P.Chevalier publie le résultat de ses observations. Opérant à la fois sur le terrain et au laboratoire, il évalue en perte de poids l'usure des blocs calcaires et des blocs en matière non soluble disposés dans le lit du torrent hypogé traversant le réseau du Trou du Glaz. La perte de poids est supérieure à l'altération chimique. Les dosages effectués au laboratoire montrent que, en moins d'une heure, la moitié du carbonate correspondant à la saturation est passé en solution la saturation étant obtenue en moins d'une dizaine d'heures. Ces mesures, à vrai dire très sommaires, confirment donc l'existence d'une corrosion limitée à la carapace lapiazée, c'est-à-dire aux quelques décamètres de la partie supérieure du massif.

En 1954, une observation de H. Roques - dissimulée dans une monographie spéléologique - renouvelait totalement la question. L'interêt et le caractère original des renseignements fournis est que l'observateur est un chimiste pur, dont les constatations paraissent difficilement contestables. En tant que géologue nous nous sommes chargé d'exploiter ces notions dans le cadre des études de spéléogenèse poursuivies depuis plusieurs années (Renault, 1960).

Dans la rivière souterraine du Saut de la Pucelle (Lot, France) les mesures effectuées montrent que, en été, les eaux sont saturées en bicarbonate dès l'entrée et la corrosion inexistante, sauf en certains lieux d'accumulation de $\mathrm{CO}_{2}$. Mais, en hiver, l'eau pénétre saturée dans la cavité, puis se réchauffe et précipite le calcaire dissous. Ceci dans la première partie de la rivière souterraine. A partir de ce point elle dissout le $\mathrm{CO}_{2}$ atmosphérique et redevient agressive.

La constatation de cette variation géochimique saisonnière explique l'opposition constatée entre les formes de surface et de profondeur à l'intérieur d'un karst.

\section{Conclusions}

Ce panorama sommaire des activités d'un laboratoire de Karstologie orienté vers l'étude de la corrosion, résume quelques tentatives ou idées, réalisées ou inspirant les travaux poursuivis dans notre laboratoire. 
Ce qui nous conduit à une remarque finale d'ordre très général.

Le praticien normal de la spéléologie a choisi cette activité en obéissant à diverses sollicitations qui n'ont rien de scientifique. Les motivations du spéléologue seront esthétiques, ou d'ordre sportif. Un certain niveau d'instruction ou de culture le poussera à décrire ce qu'il a vu sous terre, ce qui a permis l'élaboration d'une géographie spéléologique.

Mais ce domaine des cavernes doit être étudié au même titre que les grands fonds marins, la haute atmosphère ou les volcans et, de même que les alpinistes et les géologues ont parcouru indépendamment les régions de montagne, les premiers par goût personnel, les deuxièmes pour des raisons spéculatives, de même les cavernes et les régions karstiques s'offrent aux scientifiques de diverses spécialités préparés à l'étude des problèmes propres au domaine "de la roche soluble». Un géologue de montagne est aussi alpiniste et un karstologue est aussi spéléologue, mais le point de vue scientifique diffère du point de vue de l'explorateur et la découverte ne concerne plus la galerie nouvelle ou le réseau inattendu, mais le petit fait ou l'échantillon qui introduisent une théorie ou demandent une explication.

Et c'est là que le problème scientifique se pose. Tout le monde est capable de proposer une explication, c'est affaire d'imagination, mais si cette explication demeure purement verbale et fait appel à une causalité non contrôlée, elle évoque facheusement l'onde du radiesthésiste ou le magnétisme du guérisseur: un répertoire des explications toutes faites, ou passe partout, pourrait être proposé, et de nombreuses citations montreraient comment le froid, la pyrite, l'érosion, la cavitation, les tourbillons sont avancées comme évidences par des gens qui, dans le dernier cas cité par exemple, seraient incapables de définir les caractères hydrauliques d'un tourbillon, ses conditions d'apparition et sa limite d'action.

Un contrôle expérimental, et la connaissance de certaines techniques, aurait une valeur de critique, ternissant peut- être les facettes et la séduction de certaines œuvres, mais fournissant le support aride, mais solide, des modèles bien étayées.

\section{ABSTRACT}

Distinction is made between the experiment which "demonstrates" having an argumentative value - and the experiment which "questions" nature by isolating one factor and by determining the mode of its action. The concept of experiment in geology and in geodynamics and the distinctions between geodynamics and geophysics are discussed. 
Karstic geodynamics considers the action of fluids - mainly liquids - on a soluble rock. It is a science bordering the different branches of geochemistry, hydrology, the mechanics of rocks, and geophysics. Researches in karstic geodynamics are based upon measurements obtained through field surveys, or upon the utilization of a subterranean laboratory. However, in the laboratory this hardly surpasses the stage of experimental demonstration. A series of simple experiments are enumerated to exemplify the above statement, like the one where the attack of a diluted acid on a soluble rock is utilized, in order to enable us to classify the major problems encountered in karstic corrosion. The last chapter discusses the bicarbonate equilibria of $\mathrm{Ca}-\mathrm{Co}_{2}$.

Experiment furnishes the empyric criterion on which scientific theory is founded. Each discipline has its own methodology dependent on the object under study having experimental criteria of different nature. This is particularly true in case of such distant phenomena which no longer have a common ground with human dimensions like space for astronomy or time for geology. In such cases the possibilities of "instrumental" experimentations are very limited.

After a brief recollection of the principles of experimental procedure and the history of the experiments attempted by geodynamicians (tectonics, geomorphology, etc.) we will analyze several methods of investigation and by relying exactly on the example of karstic corrosion we shall determine those which have a value for the science of karstology.

\section{B I B LI OGRAPH I E}

Ajbulatov, Boldyrev, Griesseier - 1961: Das Studium der Sedimentbewegung. Petermanns Mitt., CV, 177-186.

Alexander - 1932: (Genèse des marmites de géant). J. Geol. p. 385.

Auborn, J. - 1961: Propos sur les géosynclinaux. Bull. Soc. Géol. France, (7) III, p. 630-711.

Avenard, J.M. - 1962: La solifluxion ou quelques méthodes de mécanique des sols appliquées au problème géomorphologique des versants. Tras. Lab. Géogr. Phys. Centre Géograph. Appl. Univ. Strasbourg, vol. 1, $164 \mathrm{p}$.

Balazuc, J. - 1962: Troglobies des cavités artificielles. Spelunca (4), Mém. $\mathrm{n}^{\circ} 2$, p. $104-107$.

Berthois, L. - 1962: Etude du comportement hydraulique du mica. Sedim, Amsterdam, I, n 1, p. 40-49.

Berthois, L., et Portier, J. - 1957: Recherches expérimentales sur le façonnement des graviers de quartz. C.R.Acad.Sc. France, t. 245, $\mathrm{n}^{\circ} 3$, p. 362-364.

Birot, P. - 1958: (Intervention). Bull. Assoc. Géogr. Français, p. 24.

- 1962: Contribution à l'étude de la désagrégation des roches C.D.U., Paris, $232 \mathrm{p}$.

Blanc, J. J. - 1955: In «Etude granulométrique des sédiments de la grotte du Lazaret (A.M.)" - IV - Etude physique de la sédimentation des phases argileuses et pré-colloîdales de la grotte du Lazaret. Bull. Musée Anthrop. préhist. Monaco, $\mathrm{n}^{\circ} 2$, p. 68-72. 
Bretz, J. H.-1956: Caves of Missouri. St. MissouriGéol. Surv., vol. 39, 490 p.

Capdecomme, L. - 1943: Perfectionnements récents au procédé de dosage rapide du magnésium dans les dolomies. Bull. Soc. Hist. Nat. Toulouse, t. 78 , p. $7-11$.

Garey, S. W. - 1962: Scale in geotectonic phenomena. J. Geol. India, vol. 3.

CAro, P. - 1963: La composition chimique des eaux en terrain calcaire. $5^{\circ}$ Congr. Nat. Spéléo. Millau Spelunca (4), mém. n 3, p. 39-47.

Gayeux, L. - 1930: Les roches siliceuses. Mém. Serc. Carte Géol. France, 774 p., $30 \mathrm{pl}$.

Cazalis, P. - 1961: Géomorphologie et processus expérimental. Cahiers Géogr. Québec, n 9, p. 33-50.

Chevalier, P. - 1953: Erosion ou corrosion? - $1^{\circ}$ Congr. Int. Spéléo. Paris, 1, p. 35-39.

Collin, A. - 1846: Recherches expérimentales sur les glissements spontanés des terrains argileux. Paris, $168 \mathrm{p} ., 21 \mathrm{pl}$.

Conte, P. - 1961: Géologie et phénomènes nucléaires (1ªrt.). Nucleus, $\mathrm{n}^{\circ} 3$, p. 165-172.

Gorbel, J. - 1957: Les karsts du N.O. de l'Europe. Rev. Géogr. Lyon H. S. 541 p. et 100 ph.h. t.

Cornish, V., et Kendall, P. F. - 1888: On the mineralogical constitution of calcareous organisms. Geol. mag., V, p. 66-73.

Costa de Beauregard, O. - 1963: Le second principle de la science du temps: Entropie, Information, Irréversibilité. Le Seuil éd. Paris, 158 p.

Couffignal, L. - 1963: La Cybernétique. Coll. «Que sais-je?» n638, P.U.F., Paris, $128 \mathrm{p}$.

Daubrée, A. - 1879: Etudes synthétiques de géologie expérimentale. Dunod, éd., Paris, 828 p.

Davies, W. E., et Moore, G. W. - 1957: Endellite and hydromagnesite from Carlsbad caverns. N.S.S. bull., n ${ }^{\circ} 19$, p. $24-27$.

Destouches, J. L. - 1961: In "The concept and the role of model..." Dordrecht, Hollande, $194 \mathrm{p}$.

Dor, L., et Ноттеввевx, M. - 1961: Note sur la vitesse de croissance des concrétions du type macaroni dans la grotte de Han sur Lesse. Ann. Labor. Han sur Lesse, II, ${ }^{\circ} 1$, p. 23-28.

Einstein, H. A., et Krone, R. B.-1962: Experiments to determine modes of cohesives sediments transport in salt water. J. geophys. Res. U.S.A., t. $67, \mathrm{n}^{\circ} 4$, p. $1451-1461$.

Estrem (D') - 1832: Expérience et considérations théoriques sur le mode de formation des brèches osseuses de Bise, de Cette et autres lieux voisins de la Méditerranée. Bull. Soc. Géogr. France (I), II, p. 258-259 (C. R. par Desnoyers).

Etienne, J. - 1960: Technique d'injection de résine colorée pour l'étude des roches en lame mince. Rev. Inst. Français Pétrole, t. 15, $\mathrm{n}^{\circ} 4$, p. 657-672, 32 microphotos.

Farrington, O. C. - 1901: Observations on Indiana caves. Field Columbian Mus., Publ. 53, Geol ser., vol. I, n ${ }^{\circ}$, p. 247-266.

Fouquet et Capdecoman - 1940: Sur le dosage du magnésium dans les dolomies. C. R. Acad. Sc. France, t. 211, p. 652.

Godard, A. - 1963: Deux colloques sur les processus d'altération et de pédogenèse. Rev. Géogr. Est, Nancy, France, III, nº I, p. 88-92. 
Goguet, J. - 1948: Introduction à l'étude mécanique des déformations de l'écorce terrestre. Mém. Carte Géol. Dét. France, 530 p.

— 1952: Traité de tectonique. Masson éd., Paris, 383 p.

— 1959: Préface de "La terre». Encycl. de la Pléiade. Gallimard éd., Paris.

- 1961: Géothermie. Nucleus, n 5, p. 303-308.

Grandclément, G. - 1963: L'emploi des radioéléments traçeurs en hydrologie et en hydraulique. L'eau, France, t. $50, n^{\circ} 5$, p. 293-300.

Griggs, D., et Handin, J.-1960: Rocks deformation.Geol. Soc. Americ. Mem. 382 p., $40 \mathrm{pl}$.

Haudour, J., et Sarrot Reynauld, J. - 1960: Phénomènes de dissolution du Trias dans le $\mathbf{S}$ du dôme de la Mure (Isère). C.R.somm. Soc. Géol. France, (7), II, n ${ }^{\circ}$ 8, p. 217.

Hoffmeister, J. E., et Ladd, H. S. - 1945: Solution effects on elevated limestone terraces. Bull. Geol. Soc. Americ., t. 56, p. 809-818.

HufF - 1940: voir Bretz.

KAYE, C. A. - 1957: The effect of solvent motion on limestone solution J. Geol., t. 65, n 1, p. 35-46.

KENDALL, P. F. - 1883: On the dissolution of aragonite shells in the coraline crag. Geol. Mag, X, II, 497-499.

Kolodiajnaya, A. A. - 1961: Conditions nécessaires pour la formation des composants agressifs d'eaux souterraines dans les régions karstiques (à l'exemple de l'Oural). Mém. Assoc. Int. Hydrogéol., III, p. 17-20.

Kudrin, S. N. - 1962: Essai de détermination sur le terrain des roches carbonatées à l'aide d'acide chlorhydrique à diverses concentrations. Prospection et protection du sous sol, Paris (trad. fr. du Razvedka i Okhr., russe), $\mathrm{n}^{\circ} 5$, p. 49-53.

Kuener, P. H. - 1955-1962: Experimental abrasion. Leid. geol.t. 20 (1955), p. 142-150, J. Geol. U.S., t. 64 (1956), p. 336-368, Americ. J. Sc., CCLVII (1959), p. 172-190, J. Geol. t. 68 (1960), p. 427-449, et t. 70 (1962), p. 648-658.

Lamar, J. E. - 1950: Acid etching in the study of limestones and dolomites. Cicular St.Geol. Survey Illinois, $\mathrm{n}^{\circ} 156,47 \mathrm{p}$.

Leliavsk y, S. - 1961: Précis d'hydraulique fluviale. Dunod, éd., Paris 256 p. (traduit de l'anglais).

Leopold, L. B., et LangBein, W. B. - 1962: The concept of entropy in landscape evolution. Geol. Surs. U.S.A. - Prof. Paper, n ${ }^{\circ} 500$ A, 20 p.

Loriaux, G. - 1958: De la présence de la pyrite dans les roches calcaires et de son influence dans le cavernement. Coll. Int. Spéléo., Bruxelles, Mém., p. 17-30.

Mauvisseau, J., et Renault, Рн. - 1959: La rivière souterraine d'Orchaise (Loir et Cher). Ann. Spéléo., France, t. 14, p. 81-100.

Meunier, S. - 1894: Les Causses artificiels. La Nature, Paris, t. 22, p. 243 à 246 .

- 1901: Recherches stratigraphiques et expérimentales sur la sédimentation souterraine. Assoc. Franç. Avanç. Sciences, $\mathrm{n}^{\circ}$ 29, Paris, t. 2, p. 532 à 542 .

- 1902: Stalactites de formation actuelle. La Nature, Paris, t. $30, \mathrm{n}^{\circ} 1496$, p. 121-122.

— 1902: Spéléologie expérimentale... Assoc. Franç. Avanç. Sciences, n 31 , Montauban, p. 218 et 568.

- 1904: La géologie expérimentale. Alcan, éd., Paris, 321 p. 
Mowat, G. D. - 1962: Progressive changes of shapes by solution in the laboratory. Cave notes, U.S.A., t. $4, \mathrm{n}^{\circ} 6$, p. 45-49.

Nesteroff, W. D. - 1956: Le substratum organique dans les dépôts calcaires, sa signification. Bull. Soc. Géol. France, (6), VI, p. 381-390.

Nö̈, de la, et Margerie, E. DE - 1888: Les formes du terrain. Paris.

Perna, G. - 1961 - Concrezioni in cavita artificiali. Symp. Int. Sp. Varenna, 1960. Mem. Rass. Speleo. Italiana, V. p. 225-234.

Poвeguin, T. - 1957: Reproduction expérimentale de concrétions de carbonate de calcium. Ann. Spéléo., Paris, XII, p. 5-12.

Polli, S. 1958: Meteorologia ipogea nella grotta sperimentale "C. Doria» Carso triestino. Actes $2{ }^{\circ}$ Congr. Int. Speleo. Bari, t. I, p. 449-462.

- 1958: Misure dell'accrescimento delle stalattiti. Actes $2^{\circ}$ Congr. Int. Speleo. Bari, t. I, p. 442-448.

RAmette et Heuzel - 1962: Le Rhône à Lyon. Etude de l'entrainement des galets à l'aide de traçeurs radioactifs. Houille Blanche, France 17, A, 389-399.

Renault, Ph. - 1959: Remarques sur le rôle de la pyrite dans le cavernement. Grottes et gouffres, Paris, $\mathrm{n}^{\circ} 22$, p. 17-18.

- 1960: Mise au point sur le rôle de la pyrite dans la karstification. Grottes et gouffres, Paris, $\mathrm{n}^{\circ} 23$, p. 21-24.

- 1960: Rôle de l'érosion et de la corrosion dans le creusement d'un réseau karstique. Res. Géomorph. Dyn., France, t. XI, n 1, p. 1-4.

- 1961 : Première étude météorologique de la grotte de Moulis. Ann. Spéléo., France, t. 16, p. 39-45.

— 1961: Une microforme spéléologique: les vagues d'érosion. Spelunca (4), France I, $\mathrm{n}^{\circ} 1$, p. 15-25.

- 1961: Laboratoire souterrain et spéléologie. Spelunca (4), I, n 4, p. 5-8.

- 1963: Observations récentes sur les vermiculations argileuses. Spelunca, III, $\mathrm{n}^{\circ} 1$, p. 25-28.

— 1963: Quelques réalisations de spéléologie expérimentale: vermiculations argileuses et corrosion sous remplissage. $5^{\circ}$ Congr. Nat. Spéléo. Millau, France. - Spelunica, (4), Mém. $\mathrm{n}^{\circ} 3$, p. 48-54.

Richard, E. - 1840: Pisolithe formé dans un piston de machine à vapeur. Bull. Soc. Géol. France, (I), XI, p. 228-229.

Roques, H. - depuis 1961: (Plusieurs notes conçernant les équilibres $\mathrm{CO}_{3} \mathrm{Ca}-\mathrm{CO}_{2}-\mathrm{H}_{2} \mathrm{O}$ dans les eaux karstiques). Ann. Spéléo. France.

Rougerie, G. - 1954: Méthode d'étude expérimentale des phénomènes d'érosion en milieu naturel. Res. Geomorph. Dyn., France, t. $5, \mathrm{n}^{\circ} 5$, p. 220-227.

Saporta, A. DE - 1895: Dosage du calcaire... La Nature, France, t. 23, p. 147-148.

Scheidegger, A. E. - 1961: Theoretical geomorphology. Springer éd., Berlin, $333 \mathrm{p}$.

Sмith et Albrition - 1941: Solution effects on limestone as a function of slope. Geol. Soc. Americ. Bull., t. 52, p. 61-78.

Steidtuann, E. - 1936: Humidity and waters of a limestone cavern near Lexington, Virginia. Virginia geol. sure., bull. 46 E, p. 37-45.

Talobre, J. - 1957: La mécanique des roches. Dunod, éd., Paris, 444 p. TASSY - 1843: Fragment de dépôt stalactiforme des tuyaux où circulent les eaux minérales de Chaudes Aigues (Cantal). Bull. Soc. Géol. France, (I), XIV, p. 71. 
Terzaghi, K. - 1951 : Mécanique théorique des sols. Dunod, éd., Paris, 474 p. van den Broeck, Martel, Rahir-1910: Cavernes et rivières souterraines de Belgique. Lamertin, éd., Bruxelles, 1790 p.

Vatan, A. - 1956: Travaux pratiques de pétrographie sédimentaire. Inst. français du pétrole, $207 \mathrm{p}$.

Verdeil, P. - 1958: Note préliminaire sur une classification climatique des karsts. Actes $2^{\circ}$ Congr. Int. Spéléo., Bari, t. 1, p. 49-61.

Winkler, H. G. F. et Platen, H. von - 1960-1961: Métamorphose expérimentale des roches. Geochim. Cosmochim. Acta G.B., t. 18, $\mathrm{n}^{\circ} 3-4$, p. $294-316$, t. $24, \mathrm{n}^{\circ} 1-2$, p. $48-69, \mathrm{n}^{\circ} 3-4$, p. 250-259. 